\title{
Almost Periodic Functions on the Quantum Time Scale and Applications
}

\author{
Yongkun Li (iD) \\ Department of Mathematics, Yunnan University, Kunming, Yunnan 650091, China \\ Correspondence should be addressed to Yongkun Li; yklie@ynu.edu.cn
}

Received 23 April 2019; Accepted 12 June 2019; Published 15 July 2019

Academic Editor: Allan C. Peterson

Copyright (c) 2019 Yongkun Li. This is an open access article distributed under the Creative Commons Attribution License, which permits unrestricted use, distribution, and reproduction in any medium, provided the original work is properly cited.

\begin{abstract}
In this paper, we first propose two types of concepts of almost periodic functions on the quantum time scale. Secondly, we study some basic properties of almost periodic functions on the quantum time scale. Thirdly, based on these, we study the existence and uniqueness of almost periodic solutions of dynamic equations on the quantum time scale by Lyapunov method. Then, we give an equivalent definition of almost periodic functions on the quantum time scale. Finally, as an application, we propose a class of highorder Hopfield neural networks on the quantum time scale and establish the existence and global exponential stability of almost periodic solutions of this class of neural networks.
\end{abstract}

\section{Introduction}

The concept of almost periodicity was initiated by the Bohr during the period 1923-1925 [1, 2]. Bohr's theory quickly attracted the attention of very famous mathematicians of that time. And since then the questions of the theory of almost periodic functions and almost periodic solutions of differential equations have been very interesting and challenge problems of great importance. The interaction between these two theories has enriched both. On one hand, it is well known that, in Celestial Mechanics, almost periodic solutions and stable solutions are intimately related. In the same way, stable electronic circuits exhibit almost periodic behavior. On the other hand, certain problems in differential equations have led to new definitions and results in the theory of almost periodic functions.

In recent years, the theory of quantum calculus has received much attention, due to its tremendous applications in several fields of physics, such as cosmic strings and black holes, conformal quantum mechanics, nuclear and high energy physics, fractional quantum Hall effect, and high$T_{c}$ superconductors. For interested reader, we refer to, for example, [3-6] and the references cited therein.

On one hand, recently, Bohner and Chieochan [7] introduced in the literature the concept of periodicity for functions defined on the quantum time scale owing to the fact that taking into account the periodicity of $q$-difference equations is important in order to better understand several physics phenomena. However, in reality, the almost periodic phenomenon is more common and complicated than the periodic one. Therefore, investigating the almost periodicity of dynamic equations on the quantum time scale is more interesting and more challenge.

On the other hand, in order to study the almost periodicity on time scales, a concept of almost periodic time scales was proposed in [8]. Based on this concept, a series of concepts of almost periodic function classes, such as almost periodic functions [8], pseudo almost periodic functions [9], almost automorphic functions [10], weighted pseudo almost automorphic functions [11], almost periodic set-valued functions [12], and almost periodic functions in the sense of Stepanov on time scales [13] were defined successively. Although the concept of almost periodic time scales in [8] can unify the continuous and discrete situations effectively, it is very restrictive because it requires the time scale with certain global additivity. This excludes some interesting time scales, for instance, the quantum time scale, which has no such global additivity.

Motivated by the above discussion, our main purpose of this paper is to propose two types of definitions of almost periodic functions on the quantum time scale, to study some basic properties of almost periodic functions on the quantum 
time scale, to give an equivalent definition of almost periodic functions on the quantum time scale, and to explore some of their applications to periodic dynamic equations on the quantum time scale.

The organization of this paper is as follows. In Section 2, we introduce some notations and definitions of time scale calculus. In Section 3, we propose the concepts of almost periodic functions on the quantum time scale and investigate some of their basic properties. In Section 4, we study the existence and uniqueness of almost periodic solutions of dynamic equations on the quantum time scale by Lyapunov method. In Section 5, we give an equivalent definition of almost periodic functions on the quantum time scale. In Section 6, as an application of our results, we first propose a class of high-order Hopfield neural networks on the quantum time scale; then by the exponential dichotomy of linear dynamic equations on time scales and the Banach fixed point theorem, we establish the existence and global exponential stability of almost periodic solutions of this class of neural networks. In Section 7, we draw a conclusion.

\section{Preliminaries}

In this section, we shall recall some basic definitions of time scale calculus.

A time scale $\mathbb{T}$ is an arbitrary nonempty closed subset of the real numbers, the forward and backward jump operators $\sigma, \rho: \mathbb{T} \longrightarrow \mathbb{\mathbb { }}$ and the forward graininess $\mu: \mathbb{T} \longrightarrow \mathbb{R}^{+}$are defined, respectively, by

$$
\begin{aligned}
& \sigma(t):=\inf \{s \in \mathbb{T}: s>t\}, \\
& \rho(t):=\sup \{s \in \mathbb{T}: s<t\}, \\
& \mu(t)=\sigma(t)-t .
\end{aligned}
$$

A point $t$ is said to be left-dense if $t>\inf \mathbb{T}$ and $\rho(t)=t$, right-dense if $t<\sup \mathbb{\mathbb { V }}$ and $\sigma(t)=t$, left-scattered if $\rho(t)<$ $t$, and right-scattered if $\sigma(t)>t$. If $\mathbb{T}$ has a left-scattered maximum $m$, then $\mathbb{T}^{k}=\mathbb{T} \backslash m$; otherwise $\mathbb{T}^{k}=\mathbb{T}$. If $\mathbb{T}$ has a right-scattered minimum $m$, then $\mathbb{T}_{k}=\mathbb{T} \backslash m$; otherwise $\mathbb{T}^{k}=\mathbb{T}$.

Definition 1 (see [8]). A time scale is called an almost periodic time scale if

$$
\Pi:=\{\tau \in \mathbb{R}: t+\tau \in \mathbb{T}, \forall t \in \mathbb{T}\} \neq\{0\} .
$$

A function $f: \mathbb{T} \longrightarrow \mathbb{R}$ is right-dense continuous or rdcontinuous provided it is continuous at right-dense points in $\mathbb{T}$ and its left-sided limits exist (finite) at left-dense points in $\mathbb{T}$. If $f$ is continuous at each right-dense point and each leftdense point, then $f$ is said to be a continuous function on $\mathbb{T}$.

For $f: \mathbb{T} \longrightarrow \mathbb{R}$ and $t \in \mathbb{T}^{k}$, then $f$ is called delta differentiable at $t \in \mathbb{T}$ if there exists $c \in \mathbb{R}$ such that, for given any $\varepsilon \geq 0$, there is an open neighborhood $U$ of $t$ satisfying

$$
|[f(\sigma(t))-f(s)]-c[\sigma(t)-s]| \leq \varepsilon|\sigma(t)-s|
$$

for all $s \in U$. In this case, $c$ is called the delta derivative of $f$ at $t \in \mathbb{T}$ and is denoted by $c=f^{\Delta}(t)$. For $\mathbb{T}=\mathbb{R}$, we have $f^{\Delta}=$ $f^{\prime}$, the usual derivative, for $\mathbb{T}=\mathbb{Z}$, we have the backward difference operator, $f^{\Delta}(t)=\Delta f(t):=f(t+1)-f(t)$, and, for $\mathbb{T}=\overline{q^{\mathbb{Z}}}(q>1)$, the quantum time scale, we have the $q$ derivative:

$$
f^{\Delta}(t):=D_{q} f(t)= \begin{cases}\frac{f(q t)-f(t)}{(q-1) t}, & t \neq 0, \\ \lim _{t \rightarrow 0} \frac{f q t)-f(t)}{(q-1) t}, & t=0 .\end{cases}
$$

Remark 2. Note that

$$
D_{q} f(0)=\frac{d f(0)}{d t}
$$

if $f$ is continuously differentiable.

Define for $V \in C_{r d}\left[\overline{q^{\mathbb{Z}}} \times \mathbb{R}^{n}, \mathbb{R}\right], D_{q}^{+} V^{\Delta}(t, x(t))$ to mean that, given $\varepsilon>0$, there exists a right neighborhood $N_{\varepsilon} \subset N$ such that

$$
\begin{aligned}
& \frac{1}{\mu(t, s)}[V(\sigma(t), x(\sigma(t))) \\
& -V(s, x(\sigma(t))-\mu(t, s) f(t, x(t)))] \\
& \quad<D_{q}^{+} V^{\Delta}(t, x(t))+\varepsilon
\end{aligned}
$$

for each $s \in N_{\varepsilon}, s>t$, where $\mu(t, s) \equiv \sigma(t)-s$. If $t$ is rightscattered and $V(t, x(t))$ is continuous at $t$; this reduces to

$$
D_{q}^{+} V^{\Delta}(t, x(t))=\frac{V(\sigma(t), x(\sigma(t)))-V(t, x(t))}{\sigma(t)-t} .
$$

Lemma 3 (see [14]). Let $y, f \in C_{r d}$ and $p \in \mathscr{R}^{+}$; then

$$
D_{q} y(t) \leq p(t) y(t)+f(t) \quad \text { for all } t \in \overline{q^{\mathbb{Z}}}
$$

implies

$$
y(t) \leq y\left(t_{0}\right) e_{p}\left(t, t_{0}\right)+\int_{t_{0}}^{t} e_{p}(t, \sigma(\tau)) f(\tau) d_{q} \tau
$$

$$
\text { for all } t \in \overline{q^{\mathbb{Z}}}
$$

where $t_{0} \in \overline{q^{\mathbb{Z}}}$.

For more details about the theory of time scale calculus and the theory of quantum calculus, the reader may want to consult $[3,14,15]$.

\section{Almost Periodic Functions}

From now on, we use $\mathbb{X}$ to denote the complex numbers $\mathbb{C}$ or the real numbers $\mathbb{R}$, use $D$ to denote an open set in $\mathbb{X}$ or $D=\mathbb{X}$, and use $S$ to denote an arbitrary compact subset of $D$.

Definition 4. A function $f \in C\left(\overline{q^{\mathbb{Z}}} \times D, \mathbb{X}\right)$ is called an almost periodic function in $t \in \overline{q^{\mathbb{Z}}}$ uniformly in $x \in D$ if for any 
given $\varepsilon>0$ and each compact subset $S$ of $D$; there exists $\ell(\varepsilon, S)>0$ such that each interval $\left[q^{p}, q^{p} q^{\ell}\right] \cap \overline{q^{\mathbb{Z}}}$ contains a $q^{\tau}(p, \varepsilon, S) \in\left[q^{p}, q^{p} q^{\ell}\right] \cap \overline{q^{\mathbb{Z}}}(p \in \mathbb{Z})$ such that

$$
\left|f\left(t q^{\tau}, x\right)-f(t, x)\right|<\varepsilon, \quad \forall(t, x) \in \overline{q^{\mathbb{Z}}} \times S .
$$

This $q^{\tau}$ is called the $\varepsilon$-translation number of $f$ and $\ell(\varepsilon, S)$ is called the inclusion length of the set $E\{\varepsilon, f, S\}=\left\{q^{\tau} \in \overline{q^{\mathbb{Z}}}\right.$ : $\left.\left|f\left(t q^{\tau}, x\right)-f(t, x)\right|<\varepsilon, \forall(t, x) \in \overline{q^{\mathbb{Z}}} \times S\right\}$.

Definition 5. A function $f \in C\left(\overline{q^{\mathbb{Z}}} \times D, \mathbb{X}\right)$ is called an almost periodic function in $t \in \overline{q^{\mathbb{Z}}}$ uniformly in $x \in D$ if, for any given $\varepsilon>0$ and each compact subset $S$ of $D$, there exists $\ell(\varepsilon, S)>0$ such that each interval $\left[q^{p}, q^{p} q^{\ell}\right] \cap \overline{q^{\mathbb{Z}}}$ contains a $q^{\tau}(p, \varepsilon, S) \in\left[q^{p}, q^{p} q^{\ell}\right] \cap \overline{q^{\mathbb{Z}}}(p \in \mathbb{Z})$ such that

$$
\begin{aligned}
&\left|q^{\tau} f\left(t q^{\tau}, x\right)-f(t, x)\right|<\varepsilon, \\
& \forall(t, x) \in\left(\overline{q^{\mathbb{Z}}} \backslash\{0\}\right) \times S .
\end{aligned}
$$

This $q^{\tau}$ is called the $\varepsilon$-translation number of $f$ and $\ell(\varepsilon, S)$ is called the inclusion length of the set $E\{\varepsilon, f, S\}=\left\{q^{\tau} \in \overline{q^{\mathbb{Z}}}\right.$ : $\left.\left|q^{\tau} f\left(t q^{\tau}, x\right)-f(t, x)\right|<\varepsilon, \forall(t, x) \in\left(\overline{q^{\mathbb{Z}}} \backslash\{0\}\right) \times S\right\}$.

Definition 6. A function $f \in C\left(\overline{q^{\mathbb{Z}}}, \mathbb{X}\right)$ is called an almost periodic function if, for any given $\varepsilon>0$, there exists $\ell(\varepsilon)>0$ such that each interval $\left[q^{p}, q^{p} q^{\ell}\right] \cap \overline{q^{\mathbb{Z}}}$ contains a $q^{\tau}(p, \varepsilon) \in$ $\left[q^{p}, q^{p} q^{\ell}\right] \cap \overline{q^{\mathbb{Z}}}(p \in \mathbb{Z})$ such that

$$
\left|f\left(t q^{\tau}\right)-f(t)\right|<\varepsilon, \quad \forall t \in \overline{q^{\mathbb{Z}}} .
$$

This $q^{\tau}$ is called the $\varepsilon$-translation number of $f$ and $\ell(\varepsilon)$ is called the inclusion length of the set $E\{\varepsilon, f\}=\left\{q^{\tau} \in \overline{q^{\mathbb{Z}}}\right.$ : $\left.\left|f\left(t q^{\tau}\right)-f(t)\right|<\varepsilon, \forall t \in \overline{q^{\mathbb{Z}}}\right\}$.

Definition 7. A function $f \in C\left(\overline{q^{\mathbb{Z}}}, \mathbb{X}\right)$ is called an almost periodic function if, for any given $\varepsilon>0$, there exists $\ell(\varepsilon)>0$ such that each interval $\left[q^{p}, q^{p} q^{\ell}\right] \cap \overline{q^{\mathbb{Z}}}$ contains a $q^{\tau}(p, \varepsilon) \in$ $\left[q^{p}, q^{p} q^{\ell}\right] \cap \overline{q^{\mathbb{Z}}}(p \in \mathbb{Z})$ such that

$$
\left|q^{\tau} f\left(t q^{\tau}\right)-f(t)\right|<\varepsilon, \quad \forall t \in \overline{q^{\mathbb{Z}} \backslash\{0\} .}
$$

This $q^{\tau}$ is called the $\varepsilon$-translation number of $f$ and $\ell(\varepsilon)$ is called the inclusion length of the set $E\{\varepsilon, f\}=\left\{q^{\tau} \in \overline{q^{\mathbb{Z}}}\right.$ : $\left.\left|q^{\tau} f\left(t q^{\tau}\right)-f(t)\right|<\varepsilon, \forall t \in \overline{q^{\mathbb{Z}}}\right\}$.

Definition 8. A function $f \in C\left(\overline{q^{\mathbb{Z}}}, \mathbb{E}^{n}\right)$ is called an asymptotically almost periodic function if

$$
f(t)=p(t)+q(t),
$$

where $p$ is an almost periodic function on $\overline{q^{\mathbb{Z}}}$, and $q(t) \longrightarrow 0$ as $t \longrightarrow \infty$.

For convenience, we introduce some notations.
Let $B C\left(\overline{q^{\mathbb{Z}}} \times D, \mathbb{X}\right)=\left\{f \in C\left(\overline{q^{\mathbb{Z}}} \times D, \mathbb{X}\right)\right.$ is bounded on $\left.\overline{q^{\mathbb{Z}}} \times D\right\}, A P\left(\overline{q^{\mathbb{Z}}} \times D, \mathbb{X}\right)=\left\{f \in C\left(\overline{q^{\mathbb{Z}}} \times D, \mathbb{X}\right)\right.$ is almost periodic in $t \in \overline{q^{\mathbb{Z}}}$ uniformly in $\left.x \in D\right\}$ and $A P\left(\overline{q^{\mathbb{Z}}}, \mathbb{X}\right)=\{f \in$ $C\left(\overline{q^{\mathbb{Z}}}, \mathbb{X}\right)$ is almost periodic $\}$.

Let $\alpha=\left\{\alpha_{n}\right\} \subset \mathbb{Z}$ and $\beta=\left\{\beta_{n}\right\} \subset \mathbb{Z}$ be two sequences of integer numbers. We denote $\alpha+\beta:=\left\{\alpha_{n}+\beta_{n}\right\},-\alpha:=\left\{-\alpha_{n}\right\}$ and use $\beta \subset \alpha$ to denote that $\beta$ is a subsequence of $\alpha$. We say $\alpha$ and $\beta$ are common subsequences of $\alpha^{\prime}$ and $\beta^{\prime}$, respectively, if there is some given function $n(v)$ such that $\alpha_{n}=\alpha_{n(v)}^{\prime}$ and $\beta_{n}=\beta_{n(v)}^{\prime}$.

We introduce two translation operators $T$ and $\widehat{T}$, $T_{\alpha} f(t, x)=g(t, x)$ and $\widehat{T} f(t, x)=g(t, x)$ mean that $g(t, x)=\lim _{n \rightarrow+\infty} f\left(q^{\alpha_{n}} t, x\right)$ and $g(t, x)=$ $\lim _{n \rightarrow+\infty} q^{\alpha_{n}} f\left(q^{\alpha_{n}} t, x\right)(t \quad \neq 0)$, respectively, and are written only when the limits exist. The mode of convergence will be specified at each use of the symbols.

Remark 9. When study the properties of almost periodic functions defined by Definitions 4 and 6, we use the translation operator $T$. When studying the properties of almost periodic functions defined by Definitions 5 and 7 , we use the translation operator $\bar{T}$.

Remark 10. All the results of this section hold for almost periodic functions defined by Definitions $4,5,6$, and 7 . Since their proofs are similar, we only prove them under Definitions 4 and 6.

Remark 11. A function $f: \overline{q^{\mathbb{Z}}} \times D \longrightarrow \mathbb{X}$ is continuous if and only if $\lim _{n \rightarrow-\infty} f\left(q^{n}, x\right)=f(0, x)$ exists uniformly in $x \in S$.

Theorem 12. Let $f \in A P\left(\overline{q^{\mathbb{Z}}} \times D, \mathbb{X}\right)$; then it is uniformly continuous and bounded on $\overline{q^{\mathbb{Z}}} \times S$.

Proof. For a given $\varepsilon_{0} \leq 1$ and some compact subset $S \subset D$, there exists $\ell(\varepsilon, S)$ such that, in each interval $\left[q^{p}, q^{p} q^{\ell}\right] \cap$ $\overline{q^{\mathbb{Z}}}(p \in \mathbb{Z})$, there exists a $q^{\tau} \in E\left\{\varepsilon_{0}, f, S\right\}$ such that

$$
\left|f\left(q^{\tau} t, x\right)-f(t, x)\right|<\varepsilon_{0} \leq 1, \quad \forall(t, x) \in \overline{q^{\mathbb{Z}}} \times S .
$$

It follows from $f \in C\left(\overline{q^{\mathbb{Z}}} \times D, \mathbb{X}\right)$ that, for any $(t, x) \in\left(\left[1, q^{\ell}\right] \cap\right.$ $\left.\overline{q^{\mathbb{Z}}}\right) \times S$, there exists $M>0$ such that $|f(t, x)|<M$. For any given $t \in \overline{q^{\mathbb{Z}}}$ and $t \neq 0$, take $q^{\tau} \in E(\varepsilon, f, S) \cap\left[t^{-1}, t^{-1} q^{\ell}\right]$; then $t q^{\tau} \in\left[1, q^{\ell}\right] \cap \overline{q^{\mathbb{Z}}}$. Hence, for $x \in S$, we get

$$
\begin{array}{r}
\left|f\left(t q^{\tau}, x\right)\right|<M, \\
\left|f\left(t q^{\tau}, x\right)-f(t, x)\right|<1 .
\end{array}
$$

Therefore, we obtain

$$
\begin{aligned}
|f(t, x)|<\max \left\{\max _{x \in S}|f(0, x)|, M\right\} & +1, \\
& \forall(t, x) \in \overline{q^{\mathbb{Z}}} \times S .
\end{aligned}
$$

Furthermore, because of the continuity of $f$ at $(0, x), x \in$ $S$, for any given $\varepsilon>0$, there exists a positive integer $N_{0}(\varepsilon)$ 
such that $\left|f\left(q^{n}, x\right)-f(0, x)\right|<\varepsilon / 2$ for all $n<-N_{0}$ and $x \in S$. Thus, for any $\left(q^{n_{1}}, x\right),\left(q^{n_{2}}, x\right) \in\left(\left[0, q^{-N_{0}}\right] \cap \overline{q^{\mathbb{Z}}}\right) \times S$, we find

$$
\begin{aligned}
& \left|f\left(q^{n_{1}}, x\right)-f\left(q^{n_{2}}, x\right)\right| \\
& \quad<\left|f\left(q^{n_{1}}, x\right)-f(0, x)\right|+\left|f\left(q^{n_{2}}, x\right)-f(0, x)\right| \\
& \quad<\varepsilon ;
\end{aligned}
$$

therefore, $f(t, x)$ is uniformly continuous on $\left(\left[0, q^{-N_{0}}\right] \cap \overline{q^{\mathbb{Z}}}\right) \times$ $S$. Since all the points in the interval $\left[q^{-N_{0}},+\infty\right) \cap \overline{q^{\mathbb{Z}}}$ are isolated points and $\mu(t) \geq(q-1) q^{-N_{0}}$ for all $t \in\left[q^{-N_{0}},+\infty\right) \cap$ $\overline{q^{\mathbb{Z}}}$, so $f(t, x)$ is uniformly continuous on $\left(\left[q^{-N_{0}},+\infty\right) \cap \overline{q^{\mathbb{Z}}}\right) \times$ $S$. The proof is completed.

Theorem 13. Let $f \in A P\left(\overline{q^{\mathbb{Z}}} \times D, \mathbb{X}\right)$; then, for any given sequence $\alpha^{\prime} \subset \mathbb{Z}$, there exist a subsequence $\beta \subset \alpha^{\prime}$ and $g \in C\left(\overline{q^{\mathbb{Z}}} \times D, \mathbb{X}\right)$ such that $T_{\beta} f=$ g holds uniformly on $\overline{q^{\mathbb{Z}}} \times S$ and $g \in A P\left(\overline{q^{\mathbb{Z}}} \times D, \mathbb{X}\right)$.

Proof. For any given $\varepsilon>0$ and sequence $\alpha^{\prime}=\left\{\alpha_{n}^{\prime}\right\} \subset \mathbb{Z}$, we denote $\alpha_{n}^{\prime}=\tau_{n}^{\prime}+\gamma_{n}^{\prime}$, where $q^{\tau_{n}^{\prime}} \in E\{\varepsilon / 2, f, S\}, \gamma_{n}^{\prime} \in \mathbb{Z}$, and $0 \leq \gamma_{n}^{\prime} \leq \ell(\varepsilon / 2, S), n=1,2, \ldots$. Hence, it is easy to see that there exists a subsequence $\gamma=\left\{\gamma_{n}\right\} \subset \gamma^{\prime}=\left\{\gamma_{n}^{\prime}\right\}$ such that $\gamma_{n}=s$ for $n \in \mathbb{N}, 0 \leq s \leq \ell$. We can take $\alpha \subset \alpha^{\prime}, \tau \subset \tau^{\prime}$ such that $\alpha, \tau$ common with $\gamma$ and $\alpha_{n}=\tau_{n}+s$ for all $n \in \mathbb{N}$; then

$$
\begin{aligned}
& \left|f\left(t q^{\tau_{p}-\tau_{m}}, x\right)-f(t, x)\right| \\
& \leq\left|f\left(t q^{\tau_{p}-\tau_{m}}, x\right)-f\left(t q^{\tau_{p}}, x\right)\right| \\
& \quad+\left|f\left(t q^{\tau_{p}}, x\right)-f(t, x)\right|<\varepsilon,
\end{aligned}
$$

which implies that

$$
q^{\alpha_{p}-\alpha_{m}}=q^{\tau_{p}-\tau_{m}} \in E\{\varepsilon, f, S\} .
$$

Hence, we can get

$$
\begin{aligned}
& \left|f\left(t q^{\alpha_{p}}, x\right)-f\left(t q^{\alpha_{m}}, x\right)\right| \\
& \quad \leq \sup _{(t, x) \in \overline{q^{\bar{Z}}} \times S}\left|f\left(t q^{\alpha_{p}}, x\right)-f\left(t q^{\alpha_{m}}, x\right)\right| \\
& \quad \leq \sup _{(t, x) \in \bar{q}^{\bar{Z}} \times S}\left|f\left(t q^{\alpha_{p}-\alpha_{m}}, x\right)-f(t, x)\right|<\varepsilon .
\end{aligned}
$$

Thus, we can take subsequences $\alpha^{(k)}=\left\{\alpha_{n}^{(k)}\right\}, k=1,2, \ldots$, and $\alpha^{(k+1)} \subset \alpha^{(k)} \subset \alpha$ such that, for any integers $m, p$, and all $(t, x) \in \overline{q^{\mathbb{Z}}} \times S$,

$$
\left|f\left(t q^{\alpha_{p}^{(k)}}, x\right)-f\left(t q^{\alpha_{m}^{(k)}}, x\right)\right|<\frac{1}{k}, \quad k=1,2, \ldots
$$

For all sequences $\alpha^{(k)}, k=1,2, \ldots$, we can take a sequence $\beta=\left\{\beta_{n}\right\}, \beta_{n}=\alpha_{n}^{(n)}$; then it is easy to see that, for any integers $p, m$ with $p<m$ and all $(t, x) \in \overline{q^{\mathbb{Z}}} \times S$,

$$
\left|f\left(t q^{\beta_{p}}, x\right)-f\left(t q^{\beta_{m}}, x\right)\right|<\frac{1}{p} .
$$

Therefore, $\left\{f\left(t q^{\beta_{n}}, x\right)\right\}$ converges uniformly on $\overline{q^{\mathbb{Z}}} \times S$; that is, $T_{\beta} f=g$ holds uniformly on $\overline{q^{\mathbb{Z}}} \times S$.

Next, we show that $g \in C\left(\overline{q^{\mathbb{Z}}} \times D, \mathbb{X}\right)$. Otherwise, there exists a point $\left(t_{0}, x_{0}\right) \in \overline{q^{\mathbb{Z}}} \times D$ such that $g$ is not continuous at this point. Then there exist $\varepsilon_{0}>0$ and sequences $\left\{\delta_{m}\right\} \subset \mathbb{R}^{+}$, $\left\{t_{m}\right\} \subset \overline{q^{\mathbb{Z}}},\left\{x_{m}\right\} \subset D$, where $\delta_{m}>0, \delta_{m} \longrightarrow 0$ as $m \longrightarrow+\infty$, $\left|t_{0}-t_{m}\right|+\left|x_{0}-x_{m}\right|<\delta_{m}$ and

$$
\left|g\left(t_{0}, x_{0}\right)-g\left(t_{m}, x_{m}\right)\right| \geq \varepsilon_{0} .
$$

Letting $\mathscr{X}=\left\{x_{m}\right\} \cup\left\{x_{0}\right\}$, obviously, $\mathscr{X}$ is a compact subset of $D$. Since $T_{\beta} f=g$ holds uniformly on $\overline{q^{\mathbb{Z}}} \times S$, there exists a positive integer $N=N\left(\varepsilon_{0}, \mathscr{X}\right)$ such that, for $n>N$,

$$
\left|f\left(t_{m} q^{\beta_{n}}, x_{m}\right)-g\left(t_{m}, x_{m}\right)\right|<\frac{\varepsilon_{0}}{3} \quad \forall m \in \mathbb{Z}^{+}
$$

and

$$
\left|f\left(t_{0} q^{\beta_{n}}, x_{0}\right)-g\left(t_{0}, x_{0}\right)\right|<\frac{\varepsilon_{0}}{3} .
$$

According to the uniform continuity of $f$ on $\overline{q^{\mathbb{Z}}} \times \mathscr{X}$, for sufficiently large $m$, we have

$$
\left|f\left(t_{0} q^{\beta_{n}}, x_{0}\right)-f\left(t_{m} q^{\beta_{n}}, x_{m}\right)\right|<\frac{\varepsilon_{0}}{3} .
$$

From (25)-(27), we get

$$
\left|g\left(t_{0}, x_{0}\right)-g\left(t_{m}, x_{m}\right)\right|<\varepsilon_{0},
$$

which contradicts (24). Therefore, $g$ is continuous on $\overline{q^{\mathbb{Z}}} \times D$.

Finally, for any compact subset $S \subset D$ and given $\varepsilon>0$, we take $q^{\tau} \in E\{\varepsilon, f, S\}$; then

$$
\left|f\left(t q^{\beta_{n}+\tau}, x\right)-f\left(t q^{\beta_{n}}, x\right)\right|<\varepsilon, \quad \forall(t, x) \in \overline{q^{\mathbb{Z}}} \times S .
$$

Letting $n \longrightarrow+\infty$, we have

$$
\left|g\left(t q^{\tau}, x\right)-g(t, x)\right| \leq \varepsilon, \quad \forall(t, x) \in \overline{q^{\mathbb{Z}}} \times S,
$$

which implies that $g \in A P\left(\overline{q^{\mathbb{Z}}} \times D, \mathbb{X}\right)$. This completes the proof.

Theorem 14. Letting $f \in B C\left(\overline{q^{\mathbb{Z}}} \times D, \mathbb{X}\right)$, if, for any sequence $\alpha^{\prime} \subset \mathbb{Z}$, there exists a subsequence $\alpha \subset \alpha^{\prime}$ such that $T_{\alpha} f$ exists uniformly on $\overline{q^{\mathbb{Z}}} \times S$; then $f \in A P\left(\overline{q^{\mathbb{Z}}} \times D, \mathbb{X}\right)$.

Proof (proof by contradiction). If $f \notin A P\left(\overline{q^{\mathbb{Z}}} \times D, \mathbb{X}\right)$, then there exist $\varepsilon_{0}>0$ and $S \subset D$ such that, for no matter how large $\ell>0$, we can always find an interval $\left[q^{p}, q^{p} q^{\ell}\right] \cap \overline{q^{\mathbb{Z}}}$ such that $\left[q^{p}, q^{p} q^{\ell}\right] \cap E\left\{\varepsilon_{0}, f, S\right\}=\emptyset$.

To this end, we take a number $\alpha_{1}^{\prime} \in \mathbb{Z}$ and find an interval $\left[q^{a_{1}}, q^{b_{1}}\right] \cap \overline{q^{\mathbb{Z}}}$ with $b_{1}-a_{1}>2\left|\alpha_{1}^{\prime}\right|$ and $a_{1}, b_{1} \in 2 \mathbb{Z}$ such that $\left[q^{a_{1}}, q^{b_{1}}\right] \cap E\left\{\varepsilon_{0}, f, S\right\}=\emptyset$. Next, we take $\alpha_{2}^{\prime}=(1 / 2)\left(a_{1}+b_{1}\right)$, and it is easy to see that $q^{\alpha_{2}^{\prime}-\alpha_{1}^{\prime}} \in\left[q^{a_{1}}, q^{b_{1}}\right] \cap \overline{q^{\mathbb{Z}}}$, and so $q^{\alpha_{2}^{\prime}-\alpha_{1}^{\prime}} \notin$ $E\left\{\varepsilon_{0}, f, S\right\}$; then we find an interval $\left[q^{a_{2}}, q^{b_{2}}\right] \cap \overline{q^{\mathbb{Z}}}$ with $b_{2}-a_{2}>$ 
$2\left(\left|\alpha_{1}^{\prime}\right|+\left|\alpha_{2}^{\prime}\right|\right)$ and $a_{2}, b_{2} \in 2 \mathbb{Z}$ such that $\left[q^{a_{2}}, q^{b_{2}}\right] \cap E\left\{\varepsilon_{0}, f, S\right\}=$ $\emptyset$. Next, we take $\alpha_{3}^{\prime}=(1 / 2)\left(a_{2}+b_{2}\right)$, obviously, $q^{\alpha_{3}^{\prime}-\alpha_{2}^{\prime}}, q^{\alpha_{3}^{\prime}-\alpha_{1}^{\prime}} \notin$ $E\left\{\varepsilon_{0}, f, S\right\}$. We can repeat these process again and again and can find $\alpha_{4}^{\prime}, \alpha_{5}^{\prime}, \ldots$, such that $q^{\alpha_{i}^{\prime}-\alpha_{j}^{\prime}} \notin E\left\{\varepsilon_{0}, f, S\right\}, i>j$. Thus, for any $i \neq j, i, j=1,2, \ldots$, without loss of generality, let $i>j$; we find

$$
\begin{aligned}
& \sup _{(t, x) \in \overline{q^{\mathbb{Z}} \times S}}\left|f\left(t q^{\alpha_{i}^{\prime}}, x\right)-f\left(t q^{\alpha_{j}^{\prime}}, x\right)\right| \\
& =\sup _{(t, x) \in \bar{q}^{\mathbb{Z}} \times S}\left|f\left(t q^{\alpha_{i}^{\prime}-\alpha_{j}^{\prime}}, x\right)-f(t, x)\right| \geq \varepsilon_{0} ;
\end{aligned}
$$

which implies that there is no uniformly convergent subsequence of $\left\{f\left(t q^{\alpha_{n}^{\prime}}, x\right)\right\}$ for $(t, x) \in \overline{q^{\mathbb{Z}}} \times S$. This is a contradiction. Hence, $f \in A P\left(\overline{q^{\mathbb{Z}}} \times D, \mathbb{X}\right)$. This completes the proof.

In view of Definitions 4 and 5, by Theorems 13 and 14, one can easily get the following two results.

Theorem 15. A function $f \in C\left(\overline{q^{\mathbb{Z}}} \times D, \mathbb{X}\right)$ is almost periodic in $t$ uniformly in $x \in D$, if and only if, for any given sequence $\alpha^{\prime} \subset \mathbb{Z}$, there exists a subsequence $\alpha \subset \alpha^{\prime}$ such that $T_{\alpha} f$ exists uniformly on $\overline{q^{\mathbb{Z}}} \times S$.

Theorem 16. A function $f \in C\left(\overline{q^{\mathbb{Z}}} \times D, \mathbb{X}\right)$ is almost periodic function in $t$ uniformly in $x \in D$, if and only if, for any given sequence $\alpha^{\prime} \subset \mathbb{Z}$, there exists a subsequence $\alpha \subset \alpha^{\prime}$ such that $\widehat{T}_{\alpha} f$ exists uniformly on $\overline{q^{\mathbb{Z}}} \times S$.

Theorem 17. If $f \in A P\left(\overline{q^{\mathbb{Z}}} \times D, \mathbb{X}\right)$ and $\varphi \in A P\left(\overline{q^{\mathbb{Z}}}, \mathbb{X}\right)$ with $\varphi(t) \subset S$ for all $t \in \overline{q^{\mathbb{Z}}}$. Then $f(\cdot, \varphi(\cdot)) \in A P\left(\overline{q^{\mathbb{Z}}}, \mathbb{X}\right)$.

Proof. It is easy to see that for any sequence $\alpha^{\prime} \subset \mathbb{Z}$, and there exist a subsequence $\alpha \subset \alpha^{\prime}$, and functions $\psi$ and $g$ such that $T_{\alpha} \varphi=\psi$ exists uniformly on $\overline{q^{\mathbb{Z}}}$ and $T_{\alpha} f=g$ exists uniformly on $\overline{q^{\mathbb{Z}}} \times S$, where $\psi \in A P\left(\overline{q^{\mathbb{Z}}}, \mathbb{X}\right)$ and $g \in A P\left(\overline{q^{\mathbb{Z}}} \times D, \mathbb{X}\right)$. Hence, $g$ is uniformly continuous on $\overline{q^{\mathbb{Z}}} \times S$; then, for any given $\varepsilon>0$, there exists $\delta(\varepsilon / 2)>0$ such that, for any $x_{1}, x_{2} \in$ $S$ and $\left|x_{1}-x_{2}\right|<\delta(\varepsilon / 2)$, we have

$$
\left|g\left(t, x_{1}\right)-g\left(t, x_{2}\right)\right|<\frac{\varepsilon}{2}, \quad \forall t \in \overline{q^{\mathbb{Z}}} .
$$

In addition, there exists an $N_{0}(\varepsilon)>0$ such that, for $n \geq N_{0}(\varepsilon)$,

$$
\left|f\left(t q^{\alpha_{n}}, x\right)-g(t, x)\right|<\frac{\varepsilon}{2}, \quad \forall(t, x) \in \overline{q^{\mathbb{Z}}} \times S
$$

and

$$
\left|\varphi\left(t q^{\alpha_{n}}\right)-\psi(t)\right|<\delta\left(\frac{\varepsilon}{2}\right), \quad \forall t \in \overline{q^{\mathbb{Z}}},
$$

where $\varphi\left(t q_{n}^{\alpha}\right) \subset S, \psi(t) \subset S$ for all $t \in \overline{q^{\mathbb{Z}}}$. Therefore, for $n \geq$ $N_{0}(\varepsilon)$,

$$
\begin{aligned}
& \left|f\left(t q^{\alpha_{n}}, \varphi\left(t q^{\alpha_{n}}\right)\right)-g(t, \psi(t))\right| \\
& \leq\left|f\left(t q^{\alpha_{n}}, \varphi\left(t q^{\alpha_{n}}\right)\right)-g\left(t, \varphi\left(t q^{\alpha_{n}}\right)\right)\right| \\
& \quad+\left|g\left(t, \varphi\left(t q^{\alpha_{n}}\right)\right)-g(t, \psi(t))\right|<\varepsilon,
\end{aligned}
$$

which implies that $T_{\alpha} f(t, \varphi(t))=g(t, \psi(t))$ exists uniformly on $\overline{q^{\mathbb{Z}}}$. Thus, $f(\cdot, \varphi(\cdot)) \in A P\left(\overline{q^{\mathbb{Z}}}, \mathbb{X}\right)$. The proof is complete.

Definition 18. Let $f \in B C\left(\overline{q^{\mathbb{Z}}} \times D, \mathbb{X}\right)$; then $H(f)=\{g$ : $\overline{q^{\mathbb{Z}}} \times D \longrightarrow \mathbb{X} \mid$ there exits $\alpha \subset \mathbb{Z}$ such that $T_{\alpha} f=g$ existing uniformly on $\overline{q^{\mathbb{Z}}} \times S$ \} is called the hull of $f$.

Theorem 19. $H(f)$ is compact in uniform norm if and only if $f \in A P\left(\overline{q^{\mathbb{Z}}} \times D, \mathbb{X}\right)$.

Proof. If $H(f)$ is a compact set, then, for each sequence $\alpha^{\prime} \subset$ $\mathbb{Z}$, there exists a subsequence $\alpha \subset \alpha^{\prime}$ such that $T_{\alpha} f$ exists uniformly on $\overline{q^{\mathbb{Z}}} \times D$.

Conversely, if $f \in A P\left(\overline{q^{\mathbb{Z}}} \times D, \mathbb{X}\right)$ and $\left\{g_{n}\right\} \subset H(f)$, then we can choose $\alpha^{\prime}=\left\{\alpha_{n}^{\prime}\right\}$ such that

$$
\left|f\left(t q^{\alpha_{n}^{\prime}}, x\right)-g_{n}(t, x)\right|<\frac{1}{n}, \quad \forall(t, x) \in \overline{q^{\mathbb{Z}}} \times S .
$$

So, we can find a subsequence $\alpha \subset \alpha^{\prime}$ such that $T_{\alpha} f$ exists uniformly on $\overline{q^{\mathbb{Z}}} \times D$. Let $\beta \subset \gamma=\{n\}$ such that $\beta$ and $\alpha$ are common subsequences; then

$$
\begin{aligned}
f\left(t q^{\alpha_{n}}, x\right)-g_{\beta_{n}}(t, x) & \longrightarrow 0 \\
& (n \longrightarrow \infty), \forall(t, x) \in \overline{q^{\mathbb{Z}}} \times S ;
\end{aligned}
$$

hence

$$
\begin{aligned}
\lim _{n \longrightarrow \infty} g_{\beta_{n}}(t, x)=T_{\alpha} f(t, x) \in H(f) & \\
& \forall(t, x) \in \overline{q^{\mathbb{Z}}} \times S ;
\end{aligned}
$$

therefore, $H(f)$ is a compact set. The proof is complete.

Theorem 20. If $f \in A P\left(\overline{q^{\mathbb{Z}}} \times D, \mathbb{X}\right)$, then, for any $g \in H(f)$, $H(f)=H(g)$.

Proof. For any $h \in H(g)$, there exists $\alpha^{\prime} \subset \mathbb{Z}$ such that $T_{\alpha^{\prime}} \mathcal{g}=h$ exists uniformly on $\overline{q^{\mathbb{Z}}} \times S$. Since $f \in A P\left(\overline{q^{\mathbb{Z}}} \times D, \mathbb{X}\right)$, one can extract a subsequence $\alpha \subset \alpha^{\prime}$ such that $T_{\alpha} f(t, x)=$ $\lim _{n \rightarrow \infty} f\left(t q^{\alpha_{n}}, x\right)$ exists uniformly on $\overline{q^{\mathbb{Z}}} \times S$. Because $g \in$ $H(f)$, so there exists $\alpha^{(1)} \subset \mathbb{Z}$ such that

$$
\lim _{n \longrightarrow+\infty} f\left(t q^{\alpha_{n}^{(1)}}, x\right)=g(t, x), \quad \forall(t, x) \in \overline{q^{\mathbb{Z}}} \times S ;
$$

hence,

$$
\lim _{n \longrightarrow+\infty} f\left(t q^{\alpha_{n}^{(1)}+\alpha_{n}}, x\right)=g\left(t q^{\alpha_{n}}, x\right)
$$

$$
\forall(t, x) \in \overline{q^{\mathbb{Z}}} \times S
$$

then, we can take $\beta=\left\{\beta_{n}\right\}=\left\{\alpha_{n}^{(1)}+\alpha_{n}\right\}$ such that

$$
\left|f\left(t q^{\beta_{n}}, x\right)-g\left(t q^{\alpha_{n}}, x\right)\right|<\frac{1}{n}, \quad \forall(t, x) \in \overline{q^{\mathbb{Z}}} \times S .
$$


It follows from $T_{\beta} f=T_{\alpha} g=T_{\alpha^{\prime}} g=h$ that $h \in H(f)$. Thus, $H(g) \subseteq H(f)$.

On the other hand, for any $g \in H(f)$, there exists $\alpha$ such that $T_{\alpha} f=g$ exists uniformly on $\overline{q^{\mathbb{Z}}} \times S$; hence,

$$
\begin{aligned}
\left|f\left(t q^{\alpha_{n}}, x\right)-g(t, x)\right| \longrightarrow & 0, \\
& n \longrightarrow \infty, \forall(t, x) \in \overline{q^{\mathbb{Z}}} \times S .
\end{aligned}
$$

Let $t q^{\alpha_{n}}=s$; we obtain

$$
\begin{aligned}
\left|f(s, x)-g\left(s q^{-\alpha_{n}}, x\right)\right| & \longrightarrow 0, \\
& n \longrightarrow \infty, \forall(s, x) \in \overline{q^{\mathbb{Z}}} \times S ;
\end{aligned}
$$

that is, $T_{-\alpha} g=f$ exists uniformly on $\overline{q^{\mathbb{Z}}} \times S$. Thus, $f \in H(g)$. Therefore, $H(f) \subseteq H(g)$. The proof is complete.

From Definition 18 and Theorem 20, one can easily show the following.

Theorem 21. If $f \in A P\left(\overline{q^{\mathbb{Z}}} \times D, \mathbb{X}\right)$, then, for any $g \in H(f)$, $g \in A P\left(\overline{q^{\mathbb{Z}}} \times D, \mathbb{X}\right)$.

Theorem 22. If $f, g \in A P\left(\overline{q^{\mathbb{Z}}} \times D, \mathbb{X}\right)$, then we have the following:

(a) $f+g, f g \in A P\left(\overline{q^{\mathbb{Z}}} \times D, \mathbb{X}\right)$.

(b) If inf ${ }_{t \in \overline{q^{\mathbb{Z}}}}|g(t, x)| \geq M>0$, then $f / g \in A P\left(\overline{q^{\mathbb{Z}}} \times D, \mathbb{X}\right)$.

Proof. (a) Since $f, g \in A P\left(\overline{q^{\mathbb{Z}}} \times D, \mathbb{X}\right)$, from any sequence $\left\{\alpha_{n}^{\prime}\right\} \subset \mathbb{Z}$ one can extract a subsequence $\left\{\alpha_{n}\right\}$ such that $T_{\alpha} f=\lim _{n \rightarrow \infty} f\left(t q^{\alpha_{n}}, x\right)$ exists uniformly on $\overline{q^{\mathbb{Z}}} \times S$. Then from the $\left\{\alpha_{n}\right\} \subset \mathbb{Z}$ we can extract a subsequence $\left\{\beta_{n}^{\prime}\right\}$ such that $T_{\beta^{\prime}} g=\lim _{n \rightarrow \infty} g\left(t q^{\beta_{n}^{\prime}}, x\right)$ exists uniformly on $\overline{q^{\mathbb{Z}}} \times S$. Consequently, we can extract a subsequence $\left\{\beta_{n}\right\} \subset\left\{\beta_{n}^{\prime}\right\}$ such that $T_{\beta}(f+g)=\lim _{n \rightarrow \infty}\left(f\left(t q^{\beta_{n}}, x\right)+g\left(t q^{\beta_{n}}, x\right)\right)$ and $T_{\beta}(f g)=\lim _{n \rightarrow \infty}\left(f\left(t q^{\beta_{n}}, x\right) g\left(t q^{\beta_{n}}, x\right)\right)$ exists uniformly on $\overline{q^{\mathbb{Z}}} \times S$. That is, $f+g, f g \in A P\left(\overline{q^{\mathbb{Z}}} \times D, \mathbb{X}\right)$.

(b) Because $g \in A P\left(\overline{q^{\mathbb{Z}}} \times D, \mathbb{X}\right)$, so, for any given $\varepsilon>$ 0 and each compact subset $S$ of $D$, there exists $\ell(\varepsilon, S)>0$ such that each interval $\left[q^{p}, q^{p} q^{\ell}\right] \cap \overline{q^{\mathbb{Z}}}$ contains a $q^{\tau}(p, \varepsilon, S) \in$ $\left[q^{p}, q^{p} q^{\ell}\right] \cap \overline{q^{\mathbb{Z}}}(p \in \mathbb{Z})$ such that

$$
\left|g\left(t q^{\tau}, x\right)-g(t, x)\right|<M^{2} \varepsilon, \quad \forall t \in \overline{q^{\mathbb{Z}}} \times S .
$$

Now

$$
\begin{aligned}
& \left|\frac{1}{g\left(t q^{\tau}, x\right)}-\frac{1}{g(t, x)}\right|=\frac{\left|g\left(t q^{\tau}, x\right)-g(t, x)\right|}{\left|g\left(t q^{\tau}, x\right) g(t, x)\right|} \\
& \leq \frac{\left|g\left(t q^{\tau}, x\right)-g(t, x)\right|}{M^{2}}<\varepsilon
\end{aligned}
$$

for all $(t, x) \in \overline{q^{\mathbb{Z}}} \times S$. That is, $1 / g \in A P\left(\overline{q^{\mathbb{Z}}} \times D, \mathbb{X}\right)$. Now using (a) it follows that $f / g \in A P\left(\overline{q^{\mathbb{Z}}} \times D, \mathbb{X}\right)$. The proof is complete.
Theorem 23. If $f_{n} \in A P\left(\overline{q^{\mathbb{Z}}} \times D, \mathbb{X}\right), n=1,2, \ldots$ and the sequence $\left\{f_{n}\right\}$ converges uniformly to $f$ on $\overline{q^{\mathbb{Z}}} \times S$, then $f \in$ $A P\left(\overline{q^{\mathbb{Z}}} \times D, \mathbb{X}\right)$.

Proof. For any $\varepsilon>0$, there exists sufficiently large $n_{0}$ such that for all $(t, x) \in \overline{q^{\mathbb{Z}}} \times S$,

$$
\left|f(t, x)-f_{n_{0}}(t, x)\right|<\frac{\varepsilon}{3} .
$$

Take $q^{\tau} \in E\left\{f_{n_{0}}, \varepsilon / 3, S\right\}$; then, for all $(t, x) \in \overline{q^{\mathbb{Z}}} \times S$, we have

$$
\begin{aligned}
\left|f\left(t q^{\tau}, x\right)-f(t, x)\right| \leq & \left|f\left(t q^{\tau}, x\right)-f_{n_{0}}\left(t q^{\tau}, x\right)\right| \\
& +\left|f_{n_{0}}\left(t q^{\tau}, x\right)-f_{n_{0}}(t, x)\right| \\
& +\left|f_{n_{0}}(t, x)-f(t, x)\right|<\varepsilon ;
\end{aligned}
$$

that is, $q^{\tau} \in E(f, \varepsilon, S)$. Therefore, $f \in A P\left(\overline{q^{\mathbb{Z}}} \times D, \mathbb{X}\right)$. This completes the proof.

Theorem 24. Let $f \in A P\left(\overline{q^{\mathbb{Z}}} \times D, \mathbb{X}\right)$ and $F(t, x):=$ $\int_{0}^{t} f(s, x) d_{q}$ s, then $F \in A P\left(\overline{q^{\mathbb{Z}}} \times D, \mathbb{X}\right)$ if and only if $F$ is bounded on $\overline{q^{\mathbb{Z}}} \times S$.

Proof. If $F \in A P\left(\overline{q^{\mathbb{Z}}} \times D, \mathbb{X}\right)$, then $F$ is bounded on $\overline{q^{\mathbb{Z}}} \times S$.

If $F$ is bounded, without loss of generality, we can assume that $F$ is a real-valued function. Denote

$$
G:=\sup _{(t, x) \in \overline{q^{\bar{Z}}} \times S} F(t, x)>g:=\inf _{(t, x) \in \bar{q}^{\bar{Z}} \times S} F(t, x) ;
$$

then, for any $\varepsilon>0$, there exist $t_{1}=q^{n_{1}}$ and $t_{2}=q^{n_{2}}$ such that

$$
\begin{aligned}
& F\left(t_{1}, x\right)<g+\frac{\varepsilon}{6}, \\
& F\left(t_{2}, x\right)>G-\frac{\varepsilon}{6},
\end{aligned}
$$

$\forall x \in S$.

Let $\ell=\ell\left(\varepsilon_{1}, S\right)$ be an inclusion length of $E\left(f, \varepsilon_{1}, S\right)$, where $\varepsilon_{1}=\varepsilon / 6 d, d=\left|t_{1}-t_{2}\right|$. For any $\alpha \in \overline{q^{\mathbb{Z}}}$, take $\tau \in E\left(f, \varepsilon_{1}, S\right) \cap$ $\left[\alpha t_{1}^{-1}, \alpha t_{1}^{-1} q^{\ell}\right]$. Let $s_{i}=t_{i} q^{\tau}(i=1,2)$ and $L=\ell+\left|n_{1}-n_{2}\right|$; then $s_{1}, s_{2} \in\left[\alpha, \alpha q^{L}\right] \cap \overline{q^{\mathbb{Z}}}$. For all $x \in S$, we have

$$
\begin{aligned}
F\left(s_{2}, x\right)-F\left(s_{1}, x\right)= & F\left(t_{2}, x\right)-F\left(t_{1}, x\right) \\
& -\int_{t_{1}}^{t_{2}} f(t, x) d_{q} t \\
& +\int_{t_{1} q^{\tau}}^{t_{2} q^{\tau}} f(t, x) d_{q} t \\
= & F\left(t_{2}, x\right)-F\left(t_{1}, x\right) \\
& +\int_{t_{1}}^{t_{2}}\left[f\left(t q^{\tau}, x\right)-f(t, x)\right] d_{q} t \\
> & G-g-\frac{\varepsilon}{3}-\varepsilon_{1} d=G-g-\frac{\varepsilon}{2} ;
\end{aligned}
$$


that is,

$$
\left(F\left(s_{1}, x\right)-g\right)+\left(G-F\left(s_{2}, x\right)\right)<\frac{\varepsilon}{2} .
$$

Since

$$
\begin{aligned}
& F\left(s_{1}, x\right)-g \geq 0, \\
& G-F\left(s_{2}, x\right) \geq 0,
\end{aligned}
$$

we have proved that, in any interval $\left[\alpha, \alpha q^{L}\right] \cap \overline{q^{\mathbb{Z}}}$, there exist $s_{1}$ and $s_{2}$ such that

$$
\begin{aligned}
& F\left(s_{1}, x\right)<g+\frac{\varepsilon}{2}, \\
& F\left(s_{2}, x\right)>G-\frac{\varepsilon}{2} .
\end{aligned}
$$

Now, let $\varepsilon_{2}=\varepsilon / 2 L$; we will show that if $q^{\tau} \in E\left(f, \varepsilon_{2}, S\right)$, then $q^{\tau} \in E(F, \varepsilon, S)$. In fact, for any $(t, x) \in \overline{q^{\mathbb{Z}}} \times S$, we can take $s_{1}, s_{2} \in\left[t, t q^{L}\right] \cap \overline{q^{\mathbb{Z}}}$ such that

$$
\begin{aligned}
& F\left(s_{1}, x\right)<g+\frac{\varepsilon}{2}, \\
& F\left(s_{2}, x\right)>G-\frac{\varepsilon}{2}
\end{aligned}
$$

thus, for $q^{\tau} \in E\left(f, \varepsilon_{2}, S\right)$, we have

$$
\begin{aligned}
F\left(t q^{\tau}, x\right)-F(t, x)= & F\left(s_{1} q^{\tau}, x\right)-F\left(s_{1}, x\right) \\
& +\int_{t}^{s_{1}} f(t, x) d_{q} t \\
& -\int_{t q^{\tau}}^{s_{1} q^{\tau}} f(t, x) d_{q} t \\
> & g-\left(g+\frac{\varepsilon}{2}\right) \\
& -\int_{t}^{t_{1}}\left[f\left(t q^{\tau}, x\right)-f(t, x)\right] d_{q} t \\
> & -\frac{\varepsilon}{2}-\varepsilon_{2} L=-\varepsilon
\end{aligned}
$$

and

$$
\begin{aligned}
F\left(t q^{\tau}, x\right)-F(t, x)= & F\left(s_{2} q^{\tau}, x\right)-F\left(s_{2}, x\right) \\
& +\int_{t}^{s_{2}} f(t, x) d_{q} t \\
& -\int_{t q^{\tau}}^{s_{2} q^{\tau}} f(t, x) d_{q} t<\frac{\varepsilon}{2}+\varepsilon_{2} L \\
= & \varepsilon .
\end{aligned}
$$

That is, for $q^{\tau} \in E\left(f, \varepsilon_{2}, S\right), q^{\tau} \in E(F, \varepsilon, S)$; therefore, $F \in$ $A P\left(\overline{q^{\mathbb{Z}}} \times D, \mathbb{X}\right)$. The proof is complete.

Theorem 25. If $f \in A P\left(\overline{q^{\mathbb{Z}}} \times D, \mathbb{X}\right), F(\cdot)$ is uniformly continuous on the value field of $f$; then $F \circ f \in A P\left(\overline{q^{\mathbb{Z}}} \times D, \mathbb{X}\right)$.
Proof. Since $F$ is uniformly continuous on the value field of $f$ and $f \in A P\left(\overline{q^{\mathbb{Z}}} \times D, \mathbb{X}\right)$, there exists a sequence $\alpha=\left\{\alpha_{n}\right\} \subset \mathbb{Z}$ such that

$$
\begin{aligned}
T_{\alpha}(F \circ f) & =\lim _{n \longrightarrow+\infty} F\left(f\left(t q^{\alpha_{n}}, x\right)\right) \\
& =F\left(\lim _{n \longrightarrow+\infty} f\left(t q^{\alpha_{n}}, x\right)\right)=F\left(T_{\alpha} f\right)
\end{aligned}
$$

holds uniformly on $\overline{q^{\mathbb{Z}}} \times S$. Hence, $F \circ f \in A P\left(\overline{q^{\mathbb{Z}}} \times D, \mathbb{X}\right)$. The proof is complete.

Theorem 26. A function $f \in A P\left(\overline{q^{\mathbb{Z}}} \times D, \mathbb{X}\right)$ if and only if, for every pair of sequences $\alpha^{\prime}, \beta^{\prime} \subset \mathbb{Z}$, there exist common subsequences $\alpha \subset \alpha^{\prime}, \beta \subset \beta^{\prime}$ such that

$$
T_{\alpha+\beta} f=T_{\alpha} T_{\beta} f, \quad \forall(t, x) \in \overline{q^{\mathbb{Z}}} \times S .
$$

Proof. If $f \in A P\left(\overline{q^{\mathbb{Z}}} \times D, \mathbb{X}\right)$, then, for every pair of sequences $\alpha^{\prime}, \beta^{\prime} \subset \mathbb{Z}$, there exists subsequence $\beta^{(2)} \subset \beta^{\prime}$ such that $T_{\beta^{(2)}} f=g \in A P\left(\overline{q^{\mathbb{Z}}} \times D, \mathbb{X}\right)$ uniformly holds on $\overline{q^{\mathbb{Z}}} \times S$. One can take that $\alpha^{(2)} \subset \alpha^{\prime}$ and $\alpha^{(2)}, \beta^{(2)}$ are the common subsequences of $\alpha^{\prime}, \beta^{\prime}$, respectively. Hence, there exists $\alpha^{(3)} \mathrm{C}$ $\alpha^{(2)}$ such that $T_{\alpha^{(3)}} g=h \in A P\left(\overline{q^{\mathbb{Z}}} \times D, \mathbb{X}\right)$ uniformly holds on $\overline{q^{\mathbb{Z}}} \times S$.

Similarly, one can take $\beta^{(3)} \subset \beta^{(2)}$ and $\beta^{(3)}, \alpha^{(3)}$ are the common subsequences of $\beta^{(2)}, \alpha^{(2)}$, respectively. Hence, there exist common subsequence $\alpha \subset \alpha^{(3)}, \beta \subset \beta^{(3)}$ such that $T_{\alpha+\beta} f=k \in A P\left(\overline{q^{\mathbb{Z}}} \times D, \mathbb{X}\right)$ holds uniformly on $\overline{q^{\mathbb{Z}}} \times S$. According to the above, it is easy to see that $T_{\beta} f=g$ and $T_{\alpha} g=h$ hold uniformly on $\overline{q^{\mathbb{Z}}} \times S$.

Hence, for any $\varepsilon>0$, if $n$ is sufficiently large, then, for any $(t, x) \in \overline{q^{\mathbb{Z}}} \times S$, we have

$$
\begin{aligned}
\left|f\left(t q^{\alpha_{n}+\beta_{n}}, x\right)-k(t, x)\right| & <\frac{\varepsilon}{3}, \\
\left|g(t, x)-f\left(t q^{\beta_{n}}, x\right)\right| & <\frac{\varepsilon}{3}, \\
\left|h(t, x)-g\left(t q^{\alpha_{n}}, x\right)\right| & <\frac{\varepsilon}{3} .
\end{aligned}
$$

Therefore,

$$
\begin{aligned}
|h(t, x)-k(t, x)| \leq \mid & \left|h(t, x)-g\left(t q^{\alpha_{n}}, x\right)\right| \\
& +\left|g\left(t q^{\alpha_{n}}, x\right)-f\left(t q^{\alpha_{n}+\beta_{n}}, x\right)\right| \\
& +\left|f\left(t q^{\alpha_{n}+\beta_{n}}, x\right)-k(t, x)\right|<\varepsilon
\end{aligned}
$$

holds for any $(t, x) \in \overline{q^{\mathbb{Z}}} \times S$. Since $\varepsilon>0$ is arbitrary, we have $h(t, x) \equiv k(t, x)$; that is, $T_{\alpha+\beta} f=T_{\alpha} T_{\beta} f$ holds uniformly on $\overline{q^{\mathbb{Z}}} \times S$.

On the other hand, if (58) holds, then, for any sequence $\gamma^{\prime} \subset \mathbb{Z}$, there exists subsequence $\gamma \subset \gamma^{\prime}$ such that $T_{\gamma} f$ exists uniformly on $\overline{q^{\mathbb{Z}}} \times S$. 
Now, we will prove that $f \in A P\left(\overline{q^{\mathbb{Z}}} \times D, \mathbb{X}\right)$. If this is not true, that is, $T_{\gamma} f(t, x)$ does not converge uniformly on $\overline{q^{\mathbb{Z}}} \times S$, then there exist $\varepsilon_{0}>0$ and subsequences $\alpha^{\prime} \subset \gamma, \beta^{\prime} \subset \gamma$, and $s^{\prime} . \alpha^{\prime}=\left\{\alpha_{n}^{\prime}\right\}, \beta^{\prime}=\left\{\beta_{n}^{\prime}\right\}, s=\left\{s_{n}^{\prime}\right\}$ such that

$$
\left|f\left(q^{s_{n}^{\prime}+\alpha_{n}^{\prime}}, x\right)-f\left(q^{s_{n}^{\prime}+\beta_{n}^{\prime}}, x\right)\right| \geq \varepsilon_{0}>0 .
$$

From (58) it follows that there exist common subsequences $\alpha^{(2)} \subset \alpha^{\prime}, s^{(2)} \subset s^{\prime}$ such that

$$
\begin{aligned}
T_{s^{(2)}+\alpha^{(2)}} f(t, x)=T_{s^{(2)}} T_{\alpha^{(2)}} f(t, x), & \\
& \forall(t, x) \in \overline{q^{\mathbb{Z}}} \times S .
\end{aligned}
$$

Take $\beta^{(2)} \subset \beta^{\prime}$ and $\beta^{(2)}, \alpha^{(2)}, s^{(2)}$ are common subsequences of $\beta^{\prime}, \alpha^{\prime}, s^{\prime}$, respectively, such that

$$
T_{s+\beta} f(t, x)=T_{s} T_{\beta} f(t, x), \quad \forall(t, x) \in \overline{q^{\mathbb{Z}}} \times S .
$$

Similarly, we can take $\alpha \subset \alpha^{(2)}$ satisfying $\alpha, \beta, s$ is common subsequences of $\alpha^{(2)}, \beta^{(2)}, s^{(2)}$, respectively; by (62), we have

$$
T_{s+\alpha} f(t, x)=T_{s} T_{\alpha} f(t, x), \quad \forall(t, x) \in \overline{q^{\mathbb{Z}}} \times S .
$$

Since $T_{\alpha} f(t, x)=T_{\beta} f(t, x)=T_{\gamma} f(t, x)$, by (63) and (64), for all $(t, x) \in \overline{q^{\mathbb{Z}}} \times S, T_{s+\beta} f(t, x)=T_{s+\alpha} f(t, x)$; that is, for all $(t, x) \in \overline{q^{\mathbb{Z}}} \times S, \lim _{n \rightarrow+\infty} f\left(t q^{s_{n}+\beta_{n}}, x\right)=$ $\lim _{n \rightarrow+\infty} f\left(t q^{s_{n}+\alpha_{n}}, x\right)$. Taking $t=0$, this contradicts (61). Therefore, $f \in A P\left(\overline{q^{\mathbb{Z}}} \times D, \mathbb{X}\right)$. The proof is complete.

\section{Almost Periodic Dynamic Equations on the Quantum Time Scale}

Consider the following nonlinear quantum dynamic equation:

$$
D_{q} x=f(t, x),
$$

where $f \in C\left(\overline{q^{\mathbb{Z}}} \times \mathbb{X}^{n}, \mathbb{X}^{n}\right)$.

Now, we establish existence theorem for almost periodic solution of (65) by using Theorem 20 .

Theorem 27. If $\phi$ is an asymptotically almost periodic solution of (65) on $q^{\mathbb{Z}^{+}}$, then the almost periodic part of $\phi$ is an almost periodic solution of (65).

Proof. Let $\phi=p+q$, where $p$ is the almost periodic part and $q(t) \longrightarrow 0$ as $t \longrightarrow \infty$. By Theorem 20, for any sequence $\alpha^{\prime}=\left\{\alpha_{n}^{\prime}\right\} \subset \mathbb{Z}$, there exists a subsequence $\alpha \subset \alpha^{\prime}$ such that $T_{\alpha^{\prime}} f=g$ holds uniformly on $\overline{q^{\mathbb{Z}}} \times S$ and $T_{\alpha} p=\psi$ holds uniformly on $\overline{q^{\mathbb{Z}}}$. Therefore, $T_{\alpha} \phi=T_{\alpha} p=\psi$ is an almost periodic solution of the corresponding equation in the hull

$$
D_{q} x(t)=g(t, x)
$$

on $\mathbb{T}$. Now that $T_{-\alpha} g=f$ holds uniformly on $\overline{q^{\mathbb{Z}}} \times S$ and $T_{-\alpha} \psi=p$ holds uniformly on $\overline{q^{\mathbb{Z}}}$; hence $p$ is an almost periodic solution of (65). The proof is complete.
Theorem 28. If for each $g \in H(f)$, the equation $D_{q} x(t)=$ $g(t, x(t))$ in the hull has a solution in $S$, where $S \subset \mathbb{E}^{n}$ is a compact set; then these solutions are almost periodic. In particular, system (65) has an almost periodic solution in $S$.

Proof. Let $\phi$ be a solution of $D_{q} x(t)=g(t, x(t))$ in $S$ with $g \in H(f)$. For any given sequences $\alpha^{\prime} \subset \mathbb{Z}$ and $\beta^{\prime} \subset \mathbb{Z}$, we can extract common subsequences $\alpha \subset \alpha^{\prime}$ and $\beta \subset \beta^{\prime}$ such that $T_{\alpha+\beta} f(t, x)=T_{\alpha} T_{\beta} f(t, x)$ holds uniformly on $\overline{q^{\mathbb{Z}} \times}$ $S$, and $T_{\alpha+\beta} \varphi$ and $T_{\alpha} T_{\beta} \varphi$ exist on any compact subset of $\overline{q^{\mathbb{Z}}}$. Therefore, both $T_{\alpha+\beta} \varphi(t)$ and $T_{\alpha} T_{\beta} \varphi(t)$ are solutions of the same equation in the hull

$$
D_{q} x(t)=T_{\alpha+\beta} f(t, x)
$$

in $S$. Hence, $T_{\alpha+\beta} \varphi=T_{\alpha} T_{\beta} \varphi$, so, $\varphi$ is an almost periodic solution. The proof is complete.

For system (65), we consider its product system:

$$
\begin{aligned}
& x^{\Delta}=f(t, x), \\
& y^{\Delta}=f(t, y) .
\end{aligned}
$$

Theorem 29. Suppose that there exists a Lyapunov function $V(t, x, y) \in C\left(q^{\mathbb{Z}^{+}} \times D \times D, \mathbb{R}\right)$ satisfying the following conditions:

(i) $a(|x-y|) \leq V(t, x, y) \leq b(|x-y|)$, where $a, b \in \mathscr{K}$ with $\mathscr{K}=\left\{a \in C\left(\mathbb{R}^{+}, \mathbb{R}^{+}\right): a(0)=0\right.$ and $a$ is increasing $\}$;

(ii) $\left|V\left(t, x_{1}, y_{1}\right)-v\left(t, x_{2}, y_{2}\right)\right| \leq L\left[\left|x_{1}-x_{2}\right|+y_{1}-y_{2}\right]$, where $L>0$ is a constant;

(iii) $D^{+} V_{(68)}^{\Delta}(t, x, y) \leq-c V(t, x, y)$, where $-c \in \mathscr{R}^{+}$and $c>0$.

Moreover, if there exists a solution $x(t)$ of (65) such that $x(t) \in S$ for $t \in q^{\mathbb{Z}^{+}}$, where $S \subset D$ is a compact set; then there exists a unique uniformly asymptotically stable almost periodic solution $p(t)$ of (65) in S. Furthermore, if $f(t, x)$ is periodic with period $\omega$ in $t$, then $p(t)$ is a periodic solution of (65) with period $\omega$.

Proof. Let $\alpha=\left\{\alpha_{n}\right\} \subset \mathbb{Z}$ be a sequence such that $\alpha_{n} \longrightarrow \infty$ as $n \longrightarrow \infty$ and $T_{\alpha} f=f$ uniformly on $\overline{q^{\mathbb{Z}}} \times S$. Assume that $\phi(t) \subset S$ is a solution of (65) for $t \in q^{\mathbb{Z}^{+}}$. Then $\phi\left(t q^{\alpha_{n}}\right)$ is a solution of the equation

$$
D_{q} x=f\left(t q^{\alpha_{n}}, x\right),
$$

which is also in $S$. For a given $\varepsilon>0$, choose an integer $k_{0}(\varepsilon)$ so large that if $m \geq k>k_{0}(\varepsilon)$, we have

$$
b(2 B) e_{-c}\left(\alpha_{k}, 0\right)<\frac{a(\varepsilon)}{2}
$$

and

$$
\left|f\left(t q^{\alpha_{k}}, x\right)-f\left(t q^{\alpha_{m}}, x\right)\right|<\frac{c a(\varepsilon)}{2 L},
$$


where $B$ is a positive constant such that $S \subset\{x:|x| \leq B\}$. Then it follows from (ii) and (iii) that

$$
\begin{aligned}
D^{+} & V^{\Delta}\left(t, \phi(t), \phi\left(t q^{-\alpha_{m}-\alpha_{k}}\right)\right) \\
& \leq-c V\left(t, \phi(t), \phi\left(t q^{-\alpha_{m}-\alpha_{k}}\right)\right) \\
& +L\left|f\left(t q^{\alpha_{m}-\alpha_{k}}, \phi\left(t q^{\left.\alpha_{m}-\alpha_{k}\right)}\right)-f\left(t, \phi\left(t q^{\alpha_{m}-\alpha_{k}}\right)\right)\right)\right| .
\end{aligned}
$$

In view of (71), we have

$$
\begin{aligned}
& D^{+} V^{\Delta}\left(t, \phi(t), \phi\left(t q^{-\alpha_{m}-\alpha_{k}}\right)\right) \\
& \quad \leq-c V\left(t, \phi(t), \phi\left(t q^{-\alpha_{m}-\alpha_{k}}\right)\right)+\frac{c a(\varepsilon)}{2},
\end{aligned}
$$

If $m \geq k \geq k_{0}(\varepsilon)$, by Lemma 3, condition (i), and (70), (73) implies that

$$
\begin{aligned}
& V\left(t q^{\alpha_{k}}, \phi\left(t q^{\alpha_{k}}\right), \phi\left(t q^{-\alpha_{m}}\right)\right) \\
& \leq e_{-c}\left(t q^{\alpha_{m}}, 0\right) V\left(0, \phi(0), \phi\left(q^{\alpha_{m}-\alpha_{k}}\right)\right) \\
& \quad+\frac{a(\varepsilon)}{2}\left(1-e_{-c}\left(t q^{\alpha_{k}}, 0\right)\right) \\
& \leq e_{-c}\left(t q^{\alpha_{m}}, 0\right) V\left(0, \phi(0), \phi\left(q^{\alpha_{m}-\alpha_{k}}\right)\right)+\frac{a(\varepsilon)}{2} \\
& <a(\varepsilon), \text { for } t \in q^{\mathbb{Z}^{+}} .
\end{aligned}
$$

Therefore, by condition (i), for $m \geq k \geq k_{0}$ and $t \in q^{\mathbb{Z}^{+}}$, we have

$$
\left|\phi\left(t q^{\alpha_{k}}\right)-\phi\left(t q^{\alpha_{m}}\right)\right|<\varepsilon,
$$

which implies that $\phi$ is asymptotically almost periodic. Hence, it follows from Theorem 27 that the almost periodic part $p(t) \subset S$ of $\phi$ is an almost periodic solution of system (65).

By using the Lyapunov function $V(t, x, y)$, it is easy to show that $p(t)$ is uniformly asymptotically stable and every solution remaining in $D$ approaches $p(t)$ as $t \longrightarrow \infty$, which also implies the uniqueness of $p(t)$.

In particular, if $f(t, x)$ is periodic in $t$ with period $\omega$, then $p\left(t q^{\omega}\right)$ is also a solution of (65) which remains in $S$. By the uniqueness of the solutions, we know that $p\left(t q^{\omega}\right)=p(t)$. This completes the proof.

\section{The Relationship of Almost Periodic Functions on the Quantum Time Scale and on the Integer Set}

In this section, we will give an equivalent definition of almost periodic functions on the quantum time scale $\overline{q^{\mathbb{Z}}}$. To this end, we introduce a notation $-\infty_{q}$ and stipulate $q^{-\infty_{q}}=0, t \pm$ $\left(-\infty_{q}\right)=t$ and $t>-\infty_{q}$ for any $t \in \mathbb{Z}$. Let $f \in C\left(\overline{q^{\mathbb{Z}}}, \mathbb{X}\right)$; we define a function $\tilde{f}: \mathbb{Z} \cup\left\{-\infty_{q}\right\} \longrightarrow \mathbb{X}$ by

$$
\tilde{f}(t)= \begin{cases}f\left(q^{t}\right), & t \in \mathbb{Z}, \\ f(0), & t=-\infty_{q} ;\end{cases}
$$

that is,

$$
f(t)= \begin{cases}\tilde{f}\left(\log _{q} t\right), & t \in q^{\mathbb{Z}}, \\ \lim _{t \rightarrow 0^{+}} f(t), & t=0 .\end{cases}
$$

Since $f(t)$ is right continuous at $t=0$, it is clear that the above definition is well defined.

Moreover, for $f \in C\left(\overline{q^{\mathbb{Z}}} \times \mathbb{X}, \mathbb{X}\right)$, we define a function $\tilde{f}: \mathbb{Z} \cup\left\{-\infty_{q}\right\} \times \mathbb{X} \longrightarrow \mathbb{X}$ by

$$
\tilde{f}(t, x)= \begin{cases}f\left(q^{t}, x\right), & (t, x) \in \mathbb{Z} \times \mathbb{X}, \\ f(0, x), & t=-\infty_{q}, x \in \mathbb{X}\end{cases}
$$

that is,

$$
f(t, x)= \begin{cases}\tilde{f}\left(\log _{q} t, x\right), & (t, x) \in q^{\mathbb{Z}} \times \mathbb{X}, \\ \lim _{t \rightarrow 0} f(t, x), & t=0, x \in \mathbb{X} .\end{cases}
$$

Since $f(t, x)$ is continuous at $(0, x)$, it is clear that the above definition is well defined.

Definition 30. A function $f \in C\left(\left(\mathbb{Z} \cup\left\{-\infty_{q}\right\}\right) \times \mathbb{X}, \mathbb{X}\right)$ is called almost periodic in $t \in \mathbb{Z} \cup\left\{-\infty_{q}\right\}$ uniformly in $x \in \mathbb{X}$ if for every $\varepsilon>0$ and each compact subset $S$ of $\mathbb{X}$ there exists an integer $l(\varepsilon)>0$ such that each interval of length $l(\varepsilon, S)$ contains an integer $\tau$ such that

$$
\begin{aligned}
|f(t+\tau, x)-f(t, x)|<\varepsilon, & \\
& \forall(t, x) \in\left(\mathbb{Z} \cup\left\{-\infty_{q}\right\}\right) \times S .
\end{aligned}
$$

This $\tau$ is called the $\varepsilon$-translation number or $\varepsilon$-almost period.

Definition 31. A function $f \in C\left(\mathbb{Z} \cup\left\{-\infty_{q}\right\}, \mathbb{X}\right)$ is called almost periodic if, for every $\varepsilon>0$, there exists an integer $l(\varepsilon)>0$ such that each interval of length $l(\varepsilon)$ contains an integer $\tau$ such that

$$
|f(t+\tau)-f(t)|<\varepsilon, \quad \forall t \in \mathbb{Z} \cup\left\{-\infty_{q}\right\} .
$$

This $\tau$ is called the $\varepsilon$-translation number or $\varepsilon$-almost period.

Remark 32. According to the operation rule about $-\infty_{q}, \mathbb{Z} \cup$ $\left\{-\infty_{q}\right\}$ is an almost periodic time scale under the Definition 1 and when $t=-\infty_{q}$, the inequalities (80) and (81) hold naturally. Hence, $\forall t \in \mathbb{Z} \cup\left\{-\infty_{q}\right\}$ and $\forall(t, x) \in\left(\mathbb{Z} \cup\left\{-\infty_{q}\right\}\right) \times S$ in (80) and (81) can be replaced by $\forall t \in \mathbb{Z}$ and $\forall(t, x) \in \mathbb{Z} \times S$, respectively.

Remark 33. Obviously, the almost periodic functions defined by Definitions 30 and 31 (which are defined on $\mathbb{Z} \cup\left\{-\infty_{q}\right\} \times \mathbb{X}$ ) or $\mathbb{Z} \cup\left\{-\infty_{q}\right\}$ have the same properties as the ordinary almost periodic functions defined on $\mathbb{Z} \times \mathbb{X}$ or $\mathbb{Z}$, respectively.

By Definition 4 and (78), one has the following.

Theorem 34. A function $f \in C\left(\overline{q^{\mathbb{Z}}} \times \mathbb{X}, \mathbb{X}\right)$ is almost periodic in $t \in \overline{q^{\mathbb{Z}}}$ for each $x \in \mathbb{X}$ if and only if the function $\tilde{f}(t, x)$ defined by (78) is almost periodic in $t \in \overline{q^{\mathbb{Z}}}$ for each $x \in \mathbb{X}$. 
By Definition 6 and (76), one has the following.

Theorem 35. A function $f \in C\left(\overline{q^{\mathbb{Z}}}, \mathbb{X}\right)$ is almost periodic if and only if the function $\widetilde{f}(t)$ defined by (76) is almost periodic.

Obviously, by Theorems 34 and 35 and Remark 33, all of the properties of almost periodic functions on the quantum time scale can be directly obtained from the corresponding properties of the ordinary almost periodic functions defined on $\mathbb{Z}$ or $\mathbb{Z} \times \mathbb{X}$.

\section{An Application}

Based on the equivalent definition of almost periodic functions on the quantum time scale, Remark 32, the transformations (76) and (78), we can transform the almost periodic problem of dynamic equations on the quantum time scale into the almost periodic problem of dynamic equations on almost periodic time scales. For example, consider the dynamic equation on the quantum time scale:

$$
\left.D_{q} x(t)=f\left(t, x(t), x\left(t q^{-\sigma(t)}\right)\right)\right), \quad t \in \overline{q^{\mathbb{Z}}},
$$

where $\sigma: \mathbb{Z} \longrightarrow \mathbb{Z}$ is a scalar delay function, $f \in C\left(\overline{q^{\mathbb{T}}} \times\right.$ $\left.\mathbb{E}^{2 n}, \mathbb{E}^{n}\right)$. Under transformation (78), (82) is transformed to

$$
\begin{array}{ll}
\Delta \tilde{x}(n)=F(n, \tilde{x}(n), \tilde{x}(n-\tilde{\sigma}(n))), & \\
& n \in \mathbb{Z} \cup\left\{-\infty_{q}\right\},
\end{array}
$$

and vice visa, where $\left.F(n)=(q-1) q^{n} \tilde{f}(n, \tilde{x}(n), \widetilde{x}(n-\widetilde{\sigma}(n)))\right)$.

Clearly, if $x(t)$ is a solution of (82) if and only if $\tilde{x}(n)$ is a solution of (83). Therefore, applying the theory of difference equations or the theory of almost periodic dynamic equations on time scales [8], we can first discuss the almost periodic problem of (83), and then by transformation (78) we can obtain the corresponding result about (82). Besides, since when $n=\{-\infty\}_{q}$, both sides of (83) are equal to 0 , so, if $\phi(n)$ is a solution of the equation,

$$
\Delta \tilde{x}(n)=F(n, \tilde{x}(n), \tilde{x}(n-\tilde{\sigma}(n))), \quad n \in \mathbb{Z},
$$

then

$$
\varphi(n)= \begin{cases}\phi(n), & n \in \mathbb{Z}, \\ \phi(0), & n=\{-\infty\}_{q}\end{cases}
$$

is a solution of (83).

As an application, we consider the following high-order Hopfield neural network on the quantum time scale $\overline{q^{\mathbb{\pi}}}$ :

$$
\begin{aligned}
& D_{q} x_{i}(t) \\
& =-c_{i}(t) x_{i}(t)+\sum_{j=1}^{m} a_{i j}(t) f_{j}\left(x_{j}\left(t q^{-\gamma_{i j}(t)}\right)\right) \\
& \quad+\sum_{j=1}^{m} \sum_{l=1}^{m} b_{i j l}(t) g_{j}\left(x_{j}\left(t q^{-\omega_{i j l}(t)}\right)\right) g_{l}\left(x_{l}\left(t q^{-v_{i j l}(t)}\right)\right) \\
& \quad+I_{i}(t), \quad t \in \overline{q^{\mathbb{T}}},
\end{aligned}
$$

where $i=1,2, \ldots, m, m$ corresponds to the number of units in a neural network, $x_{i}(t)$ corresponds to the state vector of the $i$ th unit at the time $t, c_{i}(t)$ represents the rate with which the $i$ th unit will reset its potential to the resting state in isolation when disconnected from the network and external inputs at time $t, a_{i j}(t), \alpha_{i j}(t)$, and $b_{i j l}(t)$ are the firstand second-order connection weights of the neural network at time $t, I_{i}(t)$ denotes the external inputs at time $t, f_{j}$ and $g_{j}$ are the activation functions of signal transmission, and $\gamma_{i j}(t), \omega_{i j l}(t), v_{i j l}(t): \mathbb{Z} \longrightarrow \mathbb{Z}^{+}$correspond to the transmission delays at time $t$.

Under transformation (78), (86) is transformed to

$$
\begin{aligned}
& \Delta \tilde{x}_{i}(n)=-(q-1) q^{n} \widetilde{c}_{i}(n) \tilde{x}_{i}(n)+(q-1) \\
& \cdot q^{n} \sum_{j=1}^{m} \widetilde{a}_{i j}(n) \widetilde{f}_{j}\left(\tilde{x}_{j}\left(n-\widetilde{\gamma}_{i j}(n)\right)\right)+(q-1) \\
& \cdot q^{n} \sum_{j=1}^{m} \sum_{l=1}^{m} \widetilde{b}_{i j l}(n) \tilde{g}_{j}\left(\tilde{x}_{j}\left(n-\widetilde{\omega}_{i j l}(n)\right)\right) \\
& \cdot \widetilde{g}_{l}\left(\tilde{x}_{l}\left(n-\widetilde{v}_{i j l}(n)\right)\right)+(q-1) q^{n} \widetilde{I}_{i}(n), \\
& n \in \mathbb{Z} \cup\left\{-\infty_{q}\right\}, i=1,2, \ldots, m
\end{aligned}
$$

and vice visa. If we take $\mathbb{T}=\mathbb{Z} \cup\left\{-\infty_{q}\right\}$, then (87) can be written as

$$
\begin{array}{r}
\tilde{x}_{i}^{\Delta}(t)=-\widehat{c}_{i}(t) \tilde{x}_{i}(t)+\sum_{j=1}^{m} \widehat{a}_{i j}(t) \tilde{f}_{j}\left(\tilde{x}_{j}\left(t-\tilde{\gamma}_{i j}(t)\right)\right) \\
+\sum_{j=1}^{m} \sum_{l=1}^{m} \widehat{b}_{i j l}(t) \widetilde{g}_{j}\left(\tilde{x}_{j}\left(t-\widetilde{\omega}_{i j l}(t)\right)\right) \\
\cdot \widetilde{g}_{l}\left(\tilde{x}_{l}\left(t-\widetilde{v}_{i j l}(t)\right)\right)+\widehat{I}_{i}(t), \\
t \in \mathbb{T}, i=1,2, \ldots, m,
\end{array}
$$

where $\widehat{c}_{i}(t)=(q-1) q^{t} \widetilde{c}_{i}(t), \widehat{a}_{i j}(t)=(q-1) q^{t} \widetilde{a}_{i j}(t), \widehat{b}_{i j l}(t)=$ $(q-1) q^{t} \widetilde{b}_{i j l}(t), \widehat{I}_{i}(t)=(q-1) q^{t} \widetilde{I}_{i}(t), t \in \mathbb{T}, i, j, l=1,2, \ldots, m$.

Remark 36. From the discussion above, we see that if (88) has an almost periodic solution $\tilde{x}=\left(\tilde{x}_{1}, \tilde{x}_{2}, \ldots, \tilde{x}_{m}\right)^{T}$, then $(86)$ has an almost periodic solution $x=\left(x_{1}, x_{2}, \ldots, x_{m}\right)^{T}$.

Now, we shall establish the existence and global exponential stability of almost periodic solutions of (88). First, for convenience, we introduce some notations. We will use $x=$ $\left(x_{1}, x_{2}, \ldots, x_{n}\right)^{T} \in \mathbb{R}^{n}$ to denote a column vector, in which the symbol $T$ denotes the transpose of vector. We let $|x|$ denote the absolute-value vector given by $|x|=\left(\left|x_{1}\right|,\left|x_{2}\right|, \ldots,\left|x_{n}\right|\right)^{T}$ and define $\|x\|=\max _{1 \leq i \leq n}\left|x_{i}\right|$.

Let $A P(\mathbb{T})=\{x \in C(\mathbb{T}, \mathbb{R}): x(t)$ is a real valued, almost periodic function on $\mathbb{T}\}$ with the norm $\|\varphi\|_{0}=\sup _{t \in \mathbb{T}}\|\varphi(t)\|_{0}$, where $\|\varphi(t)\|_{0}=\max _{1 \leq i \leq n}\left|\varphi_{i}(t)\right|$ and $\varphi \in A P(\mathbb{T})$; then $A P(\mathbb{T})$ is an Banach space. 
Theorem 37. Assume the following:

$\left(H_{1}\right) \widetilde{f}_{j}, \tilde{g}_{j} \in C(\mathbb{R}, \mathbb{R})$, and there exist positive constants $\epsilon_{j}$ and $\varepsilon_{j}$ such that

$$
\begin{aligned}
& \left|\tilde{f}_{j}(u)-\tilde{f}_{j}(v)\right| \leq \epsilon_{j}|u-v|, \\
& \left|\tilde{g}_{j}(u)-\tilde{g}_{j}(v)\right| \leq \varepsilon_{j}|u-v|,
\end{aligned}
$$

where $|u|,|v| \in \mathbb{R}, j=1,2, \ldots, m$;

$\left(H_{2}\right)$ there exist constants $N_{j}>0$ such that $\left|\widetilde{g}_{j}(u)\right| \leq$ $N_{j}, u \in \mathbb{R}, j=1,2, \ldots, m$;

$\left(H_{3}\right)$ for $i, j, l=1,2, \ldots, m, \widehat{c}_{i}$ is positive regressive and $\inf _{t \in \mathbb{T}} \widehat{c}_{i}(t)>0, \widehat{c}_{i}, \widehat{a}_{i j}, \widehat{b}_{i j l}, \widehat{I}_{i}, \widetilde{\gamma}_{i j}, \widetilde{\omega}_{i j l}, \widetilde{v}_{i j l} \in A P(\mathbb{T}) ;$

$\left(H_{4}\right)$ there exists a constant $r_{0}$ such that

$$
\begin{aligned}
\max _{1 \leq i \leq m}\left\{\frac{\eta_{i}}{\widehat{c}_{i}}\right\}+L \leq r_{0}, \\
\max _{1 \leq i \leq m}\left\{\bar{\eta}_{i}\right\}<\min _{1 \leq i \leq m}\left\{\widehat{c}_{i}^{-}\right\},
\end{aligned}
$$

where, for $i, j, l=1,2, \ldots, n$,

$$
\begin{aligned}
\eta_{i} & =\sum_{j=1}^{m}\left[\widehat{a}_{i j}^{+}\left(\left|f_{j}(0)\right|+\epsilon_{j} r_{0}\right)\right) \\
& \left.+\left(\left|g_{j}(0)\right|+\varepsilon_{j} r_{0}\right) \sum_{l=1}^{m} \widehat{b}_{i j l}^{+}\left(\left|g_{l}(0)\right|+\varepsilon_{l} r_{0}\right)\right], \\
\bar{\eta}_{i} & =\sum_{j=1}^{m} \widehat{a}_{i j}^{+} \epsilon_{j}+\sum_{j=1}^{m} \sum_{l=1}^{m} \widehat{b}_{i j l}^{+}\left(N_{l} \varepsilon_{j}+N_{j} \varepsilon_{l}\right), \\
L & =\max _{1 \leq i \leq n} \frac{\bar{I}_{i}}{\widehat{c}_{i}^{-}}, \\
\widehat{c}_{i}^{+} & =\sup _{t \in \mathbb{\mathbb { T }}} c_{i}(t), \\
\widehat{c}_{i}^{-} & =\inf _{t \in \mathbb{T}} \widehat{c}_{i}(t), \\
\widehat{a}_{i j}^{+} & =\sup _{t \in \mathbb{T}}\left|\widehat{a}_{i j}(t)\right|, \\
\widehat{b}_{i j l}^{+} & =\sup _{t \in \mathbb{\mathbb { T }}}\left|\widehat{b}_{i j l}(t)\right|, \\
\widehat{I}_{i}^{+} & =\sup _{t \in \mathbb{T}}\left|\widehat{I}_{i}(t)\right| .
\end{aligned}
$$

Then system (88) has a unique almost periodic solution in the region

$$
E=\left\{\varphi \in A P(\mathbb{\mathbb { T }}):\|\varphi\|_{0} \leq r_{0}\right\}
$$

Proof. For any given $\varphi \in A P(\mathbb{\mathbb { T }})$, we consider the following almost dynamic equation:

$$
\begin{array}{r}
\tilde{x}_{i}^{\Delta}(t)=-\widehat{c}_{i}(t) \tilde{x}_{i}(t)+\sum_{j=1}^{m} \widehat{a}_{i j}(t) \tilde{f}_{j}\left(\varphi_{j}\left(t-\widetilde{\gamma}_{i j}(t)\right)\right) \\
+\sum_{j=1}^{m} \sum_{l=1}^{m} \widehat{b}_{i j l}(t) \widetilde{g}_{j}\left(\varphi_{j}\left(t-\widetilde{\omega}_{i j l}(t)\right)\right) \\
\cdot \widetilde{g}_{l}\left(\varphi_{l}\left(t-\widetilde{v}_{i j l}(t)\right)\right)+\widehat{I}_{i}(t), \\
i=1,2, \ldots, m .
\end{array}
$$

In view of $\left(\mathrm{H}_{3}\right)$, it follows from Lemma 2.15 in [16] that the linear system

$$
\tilde{x}_{i}^{\Delta}(t)=-\widehat{c}_{i}(t) \tilde{x}_{i}(t), \quad i=1,2, \ldots, m
$$

admits an exponential dichotomy on $\mathbb{T}$. Thus, by Theorem 4.19 in [8], we know that system (93) has exactly one almost periodic solution

$$
\begin{aligned}
& \left(\tilde{x}_{\varphi}\right)_{i}(t)=\int_{-\infty}^{t} e_{-\widehat{c}_{i}}(t, \sigma(s))\left(\sum _ { j = 1 } ^ { m } \left[\widehat{a}_{i j}(s)\right.\right. \\
& \cdot \widetilde{f}_{j}\left(\varphi_{j}\left(s-\widetilde{\gamma}_{i j}(s)\right)\right)+\sum_{j=1}^{m} \sum_{l=1}^{m} \widehat{b}_{i j l}(s) \widetilde{g}_{j} \\
& \left.\cdot\left(\varphi_{j}\left(s-\widetilde{\omega}_{i j l}(s)\right)\right) \widetilde{g}_{l}\left(\varphi_{l}\left(s-\widetilde{v}_{i j l}(s)\right)\right)+\widehat{I}_{i}(s)\right] \\
& \cdot \Delta s, \quad i=1,2, \ldots, m .
\end{aligned}
$$

First, we define a nonlinear operator on $A P(\mathbb{T})$ by

$$
\Phi(\varphi)(t)=\tilde{x}_{\varphi}(t), \quad \forall \varphi \in A P(\mathbb{T})
$$


Next, we check that $\Phi(E) \subset E$. For any given $\varphi \in E$, it suffices to prove that $\|\Phi(\varphi)\|_{0} \leq r_{0}$. By conditions $\left(H_{1}\right)-\left(H_{4}\right)$, we have

$$
\begin{aligned}
& \|\Phi(\varphi)\|_{0} \\
& =\sup _{t \in \mathbb{T}} \max _{1 \leq i \leq m}\left\{\mid \int_{-\infty}^{t} e_{-\widehat{c}_{i}}(t, \sigma(s))\left(\sum_{j=1}^{m} \widehat{a}_{i j}(s) \tilde{f}_{\}}\right.\right. \\
& \cdot\left(\varphi_{j}\left(s-\widetilde{\gamma}_{i j}(s)\right)\right)+\sum_{j=1}^{m} \sum_{l=1}^{m} \widehat{b}_{i j l}(s) \\
& \cdot \tilde{g}_{j}\left(\varphi_{j}\left(s-\widetilde{\omega}_{i j l}(s)\right)\right) \tilde{g}_{l}\left(\varphi_{l}\left(s-\widetilde{v}_{i j l}(s)\right)\right) \\
& \left.\left.+\widehat{I}_{i}(s)\right) \Delta s \mid\right\} \\
& \leq \sup _{t \in \mathbb{N}} \max _{1 \leq i \leq m}\left\{\mid \int_{-\infty}^{t} e_{-\widehat{c}_{i}}(t, \sigma(s))\left(\sum_{j=1}^{m} \widehat{a}_{i j}^{+} \tilde{f}_{j}\right.\right. \\
& \cdot\left(\varphi_{j}\left(s-\tilde{\gamma}_{i j}(s)\right)\right)+\sum_{j=1}^{m} \sum_{l=1}^{m} \hat{b}_{i j}^{+} \tilde{l}_{j} \\
& \left.\left.\cdot\left(\varphi_{j}\left(s-\widetilde{\omega}_{i j l}(s)\right)\right) \tilde{g}_{l}\left(\varphi_{l}\left(s-\widetilde{v}_{i j l}(s)\right)\right)\right) \Delta s \mid\right\} \\
& +\max _{1 \leq i \leq n} \frac{\widehat{I_{i}^{+}}}{\widehat{c}_{i}} \\
& \leq \sup _{t \in \mathbb{T}} \max _{\mathbb{1} \leq i \leq m}\left\{\mid \int_{-\infty}^{t} e_{-\widehat{c}_{i}^{-}}(t, \sigma(s))\left(\sum_{j=1}^{m} \hat{a}_{i j}^{+}\right.\right. \\
& \cdot\left(\left|\tilde{f}_{j}(0)\right|+\epsilon_{j}\left|\varphi_{j}\left(s-\tilde{\gamma}_{i j}(s)\right)\right|\right)+\sum_{j=1}^{m} \sum_{l=1}^{m} \hat{b}_{i j l}^{+} \\
& \left.\left.\cdot\left(\left|\widetilde{g}_{j}(0)\right|+\varepsilon_{j}\left|\varphi_{j}\left(s-\widetilde{\omega}_{i j l}(s)\right)\right|\right)\left(\left|\widetilde{g}_{l}(0)\right|+\varepsilon_{l}\left|\varphi_{l}\left(s-\widetilde{v}_{i j l}(s)\right)\right|\right)\right) \Delta s \mid\right\} \\
& +L \\
& \leq \sup _{t \in \mathbb{T}} \max _{1 \leq i \leq m}\left\{\mid \int_{-\infty}^{t} e_{-\widehat{c}_{i}^{-}}(t, \sigma(s))\left(\sum_{j=1}^{m} \hat{a}_{i j}^{+}\right.\right. \\
& \cdot\left(\left|\tilde{f}_{j}(0)\right|+\epsilon_{j} r_{0}\right)+\sum_{j=1}^{m} \sum_{l=1}^{m} \hat{b}_{i j l}^{+} \\
& \left.\left.\cdot\left(\left|\widetilde{g}_{j}(0)\right|+\varepsilon_{j} r_{0}\right)\left(\mid \widetilde{g}_{l}(0)+\varepsilon_{l} r_{0}\right)\right) \Delta s \mid\right\}+L \\
& \leq \max _{1 \leq i \leq m}\left\{\frac{\eta_{i}}{\bar{c}_{i}}\right\}+L \leq r_{0}
\end{aligned}
$$

which implies that $\Phi(E) \subset E$. For any $\varphi, \psi \in E$, according to $\left(H_{1}\right)$ and $\left(H_{4}\right)$, we have

$$
\begin{aligned}
& \|\Phi(\varphi)-\Phi(\psi)\|_{0} \\
& =\sup _{t \in \mathbb{\mathbb { N }} 1 \leq i \leq m}\left\{\mid \int_{-\infty}^{t} e_{-\widehat{c}_{i}}(t, \sigma(s))\left(\sum_{j=1}^{m} \widehat{a}_{i j}(s)\right.\right. \\
& \cdot\left[\tilde{f}_{j}\left(\varphi_{j}\left(s-\widetilde{\gamma}_{i j}(s)\right)\right)-\tilde{f}_{j}\left(\psi_{j}\left(s-\widetilde{\gamma}_{i j}(s)\right)\right)\right]
\end{aligned}
$$

$$
\begin{aligned}
& +\sum_{j=1}^{m} \sum_{l=1}^{m} \widehat{b}_{i j l}(s) \widetilde{g}_{j}\left(\varphi_{j}\left(s-\widetilde{\omega}_{i j l}(s)\right)\right) \tilde{g}_{l}\left(\varphi_{l}\left(s-\widetilde{v}_{i j l}(s)\right)\right) \\
& -\sum_{j=1}^{m} b_{i j l}(s) \widetilde{g}_{j}\left(\psi_{j}\left(s-\widetilde{\omega}_{i j l}(s)\right)\right) \widetilde{g}_{l}\left(\psi_{l}\left(s-\widetilde{v}_{i j l}(s)\right)\right) \\
& \leq \sup _{t \in \mathbb{T}} \max _{\leq i \leq m}\left\{\int _ { - \infty } ^ { t } e _ { - \widetilde { c } _ { i } } ( t , \sigma ( s ) ) \left(\sum_{j=1}^{m} \widehat{a}_{i j}^{+} \epsilon_{j}\right.\right. \\
& \cdot\left|\varphi_{j}\left(s-\widetilde{\gamma}_{i j}(s)\right)-\psi_{j}\left(s-\widetilde{\gamma}_{i j}(s)\right)\right|+\sum_{j=1}^{m} \sum_{l=1}^{m} \widehat{b}_{i j l}^{+} \varepsilon_{j} \\
& \cdot\left|\varphi_{j}\left(s-\widetilde{\omega}_{i j l}(s)\right)-\psi_{j}\left(s-\widetilde{\omega}_{i j l}(s)\right)\right| \\
& \cdot\left|\widetilde{g}_{l}\left(\varphi_{l}\left(s-\widetilde{v}_{i j l}(s)\right)\right)\right|+\sum_{j=1}^{m} \sum_{l=1}^{m} \widehat{b}_{i j l}^{+} \varepsilon_{l} \mid \varphi_{l}\left(s-\widetilde{v}_{i j l}(s)\right) \\
& \left.\left.-\psi_{l}\left(s-\widetilde{v}_{i j l}(s)\right)|| \widetilde{g}_{j}\left(\psi_{j}\left(s-\widetilde{\omega}_{i j l}(s)\right)\right) \mid\right) \Delta s\right\} \\
& \leq \sup _{t \in \mathbb{T} l \leq i \leq m}\left\{\int _ { - \infty } ^ { t } e _ { - \widetilde { c } _ { i } } ( t , \sigma ( s ) ) \left(\sum_{j=1}^{m} \widehat{a}_{i j}^{+} \epsilon_{j}+\sum_{j=1}^{m} \sum_{l=1}^{m} \hat{b}_{i j l}^{+}\right.\right. \\
& \left.\left.\cdot\left(N_{j} \varepsilon_{l}+N_{l} \varepsilon_{j}\right)\right)\|\varphi-\psi\|_{0} \Delta s\right\} \\
& \leq \frac{\max _{1 \leq i \leq m}\left\{\bar{\eta}_{i}\right\}}{\min _{1 \leq i \leq m}\left\{\widetilde{c}_{i}^{-}\right\}} \| \varphi \\
& -\|_{0},
\end{aligned}
$$

which implies that $\Phi$ is a contraction mapping. Hence, $\Phi$ has a unique fixed point in $E$; that is, (88) has a unique almost periodic solution in the region $E$. This completes the proof.

Theorem 38. Assume that $\left(H_{1}\right)-\left(H_{4}\right)$ hold; then system (88) has a unique almost periodic solution that is globally exponentially stable.

Proof. From Theorem 37, we see that system (88) has a unique almost periodic solution $\widetilde{x}^{*}(t)=\left(\widetilde{x}_{1}^{*}(t), \widetilde{x}_{2}^{*}(t), \ldots, \widetilde{x}_{m}^{*}(t)\right)^{T}$ with initial value $\varphi^{*}(t)=\left(\varphi_{1}^{*}(t), \varphi_{2}^{*}(t), \ldots, \varphi_{m}^{*}(t)\right)^{T}$. Suppose that $\widetilde{x}(t)=\left(\widetilde{x}_{1}(t), \widetilde{x}_{2}(t), \ldots, \widetilde{x}_{m}(t)\right)^{T}$ is an arbitrary solution of (88) with initial value $\varphi(t)=\left(\varphi_{1}(t), \varphi_{2}(t), \ldots, \varphi_{m}(t)\right)^{T}$. Then it follows from system (88) that

$$
\begin{aligned}
& \widetilde{z}_{i}^{\Delta}(t)=-\widehat{c}_{i}(t) \tilde{z}_{i}(t)+\sum_{j=1}^{m} \widehat{a}_{i j}(t)\left(\tilde { f } _ { j } \left(\tilde{z}_{j}\left(t-\widetilde{\gamma}_{i j}(t)\right)\right.\right. \\
& \left.\left.+\tilde{x}_{j}^{*}\left(t-\tilde{\gamma}_{i j}(t)\right)\right)-\tilde{f}_{j}\left(\tilde{x}_{j}^{*}\left(t-\tilde{\gamma}_{i j}(t)\right)\right)\right) \\
& +\sum_{j=1}^{m} \sum_{l=1}^{m} \widehat{b}_{i j l}(t)
\end{aligned}
$$




$$
\begin{aligned}
& \cdot\left(\left[\tilde{g}_{j}\left(\widetilde{z}_{j}\left(t-\widetilde{\omega}_{i j l}(t)\right)+\widetilde{x}_{j}^{*}\left(t-\widetilde{\omega}_{i j l}(t)\right)\right)\right.\right. \\
& \left.-\widetilde{g}_{j}\left(\widetilde{x}_{j}^{*}\left(t-\widetilde{\omega}_{i j l}(t)\right)\right)\right] \times \widetilde{g}_{l}\left(\widetilde{z}_{l}\left(t-\widetilde{v}_{i j l}(t)\right)\right. \\
& \left.+\widetilde{x}_{l}^{*}\left(t-\widetilde{v}_{i j l}(t)\right)\right) \\
& +\left[\widetilde{g}_{l}\left(\widetilde{z}_{l}\left(t-\widetilde{v}_{i j l}(t)\right)+\widetilde{x}_{l}^{*}\left(t-\widetilde{v}_{i j l}(t)\right)\right)\right. \\
& \left.\left.-\widetilde{g}_{l}\left(\widetilde{x}_{l}^{*}\left(s-\widetilde{v}_{i j l}(t)\right)\right)\right] \widetilde{g}_{j}\left(\widetilde{x}_{j}^{*}\left(t-\widetilde{\omega}_{i j l}(t)\right)\right)\right),
\end{aligned}
$$

where $\widetilde{z}_{i}(t)=\widetilde{x}_{i}(t)-\widetilde{x}_{i}^{*}(t), i=1,2, \ldots, m$.

The initial condition of (99) is

$$
\phi_{i}(s)=\varphi_{i}(s)-\varphi_{i}^{*}(s), \quad s \in[-\tau, 0]_{\mathbb{T}}
$$

where $\tau=\max \{\widetilde{\gamma}, \widetilde{\omega}, \widetilde{v}\}, \widetilde{\gamma}=\max _{1 \leq i, j \leq m}\left\{\sup _{t \in \mathbb{T}}\left|\widetilde{\gamma}_{i j}(t)\right|\right\}, \widetilde{\omega}=$ $\max _{1 \leq i, j, l \leq m}\left\{\sup _{t \in \mathbb{T}}\left|\widetilde{\omega}_{i j l}(t)\right|\right\}, \widetilde{v}=\max _{1 \leq i, j, l \leq m}\left\{\sup _{t \in \mathbb{T}}\left|\widetilde{v}_{i j l}(t)\right|\right\}$, and $\phi_{i}(\cdot)$ denotes a real-valued bounded $\Delta$-differentiable function defined on $[-\tau, 0]_{\mathbb{T}}$.

Multiplying both sides of (99) by $e_{-\widehat{c}_{i}}\left(t_{0}, \sigma(t)\right)$ and integrating over $\left[t_{0}, t\right]_{\mathbb{T}}$, where $t_{0} \in[-\tau, 0]_{\mathbb{T}}$, for $i=1,2, \ldots, m$, we have

$$
\begin{aligned}
& \widetilde{z}_{i}(t)=\widetilde{z}_{i}\left(t_{0}\right) e_{-\widehat{c}_{i}}\left(t, t_{0}\right)+\int_{t_{0}}^{t} e_{-\widehat{c}_{i}}(t, \sigma(s))\left(\sum_{j=1}^{m} \widehat{a}_{i j}(s)\right. \\
& \cdot\left(\tilde{f}_{j}\left(\tilde{z}_{j}\left(s-\tilde{\gamma}_{i j}(s)\right)+\tilde{x}_{j}^{*}\left(s-\tilde{\gamma}_{i j}(s)\right)\right)\right. \\
& \left.-\tilde{f}_{j}\left(\tilde{x}_{j}^{*}\left(s-\widetilde{\gamma}_{i j}(s)\right)\right)\right)+\sum_{j=1}^{m} \sum_{l=1}^{m} \widehat{b}_{i j l}(s) \\
& \cdot\left(\left[\widetilde{g}_{j}\left(\widetilde{z}_{j}\left(s-\widetilde{\omega}_{i j l}(s)\right)+\widetilde{x}_{j}^{*}\left(s-\widetilde{\omega}_{i j l}(s)\right)\right)\right.\right. \\
& \left.-\widetilde{g}_{j}\left(\tilde{x}_{j}^{*}\left(s-\widetilde{\omega}_{i j l}(s)\right)\right)\right] \widetilde{g}_{l}\left(\widetilde{z}_{l}\left(s-\widetilde{v}_{i j l}(s)\right)\right. \\
& \left.+\widetilde{x}_{l}^{*}\left(s-\widetilde{v}_{i j l}(s)\right)\right) \\
& +\left[\widetilde{g}_{l}\left(\widetilde{z}_{l}\left(s-\widetilde{v}_{i j l}(s)\right)+\tilde{x}_{l}^{*}\left(s-\widetilde{v}_{i j l}(s)\right)\right)\right. \\
& \left.\left.\left.-\widetilde{g}_{l}\left(\widetilde{x}_{l}^{*}\left(s-\widetilde{v}_{i j l}(s)\right)\right)\right] \widetilde{g}_{j}\left(\widetilde{x}_{j}^{*}\left(s-\widetilde{\omega}_{i j l}(s)\right)\right)\right)\right) \Delta s .
\end{aligned}
$$

For $i=1,2, \ldots, m$, let $\Gamma_{i}$ be defined as follows:

$$
\begin{aligned}
\Gamma_{i}(\zeta) & =\widehat{c}_{i}^{-}-\zeta-\exp \left(\zeta \sup _{s \in \mathbb{T}} \mu(s)\right)\left(\sum_{j=1}^{m} \widehat{a}_{i j}^{+} \epsilon_{j} \exp (\zeta \widetilde{\gamma})\right. \\
+ & \left.\sum_{j=1}^{m} \sum_{l=1}^{m} \hat{b}_{i j l}^{+}\left[\varepsilon_{j} N_{l} \exp (\zeta \widetilde{\omega})+\varepsilon_{l} N_{j} \exp (\zeta \widetilde{v})\right]\right)
\end{aligned}
$$

By $\left(H_{4}\right)$, we get

$$
\Gamma_{i}(0)=\widehat{c}_{i}^{-}-\bar{\eta}_{i}>0, \quad i=1,2, \ldots, m .
$$

Since $\Gamma_{i}$ is continuous on $[0,+\infty)$ and $\Gamma_{i}(\zeta) \longrightarrow-\infty$ as $\gamma \longrightarrow+\infty$, there exists $\xi_{i}>0$ such that $\Gamma_{i}\left(\xi_{i}\right)=0$ and
$\Gamma_{i}(\zeta)>0$ for $\zeta \in\left(0, \xi_{i}\right)$. Take $\beta=\min _{1 \leq i \leq m}\left\{\xi_{i}\right\}$; we have $\Gamma_{i}(\beta) \geq 0$. Hence, we can choose a positive constant $0<\lambda<$ $\min \left\{\beta, \min _{1 \leq i \leq m}\left\{\widehat{c}_{i}^{-}\right\}\right\}$such that

$$
\Gamma_{i}(\lambda)>0, \quad i=1,2, \ldots, m,
$$

which implies that

$$
\begin{array}{r}
\frac{\widehat{c}_{i}^{+} \exp \left(\lambda \sup _{s \in \mathbb{T}} \mu(s)\right)}{\widehat{c}_{i}^{-}-\lambda}\left(\sum_{j=1}^{m} \widehat{a}_{i j}^{+} \epsilon_{j} \exp (\lambda \widetilde{\gamma})\right. \\
\left.+\sum_{j=1}^{m} \sum_{l=1}^{m} \widehat{b}_{i j l}^{+}\left[\varepsilon_{j} N_{l} \exp (\lambda \widetilde{\omega})+\varepsilon_{l} N_{j} \exp (\lambda \widetilde{v})\right]\right)<1, \\
i=1,2, \ldots, m .
\end{array}
$$

Taking

M

$$
=\max _{1 \leq i \leq m}\left\{\frac{\widehat{c}_{i}^{-}}{\sum_{j=1}^{m} \widehat{a}_{i j}^{+} \epsilon_{j}+\sum_{j=1}^{m} \sum_{l=1}^{m} \widehat{b}_{i j l}^{+}\left[\varepsilon_{j} N_{l}+\varepsilon_{l} N_{j}\right]}\right\},
$$

then by $\left(H_{4}\right)$ we have $M>1$. Besides, we can obtain that

$$
\begin{aligned}
\frac{1}{M}< & <\frac{\widehat{c}_{i}^{+} \exp \left(\lambda \sup _{s \in \mathbb{T}} \mu(s)\right)}{\widehat{c}_{i}^{-}-\lambda}\left(\sum_{j=1}^{m} \widehat{a}_{i j}^{+} \epsilon_{j} \exp (\lambda \widetilde{\gamma})\right. \\
& \left.+\sum_{j=1}^{m} \sum_{l=1}^{m} \widehat{b}_{i j l}^{+}\left[\varepsilon_{j} N_{l} \exp (\lambda \widetilde{\omega})+\varepsilon_{l} N_{j} \exp (\lambda \widetilde{v})\right]\right) .
\end{aligned}
$$

Let $\|\bar{z}(t)\|=\max _{1 \leq i \leq m}\left|\widehat{z}_{i}(t)\right|, \quad\|\phi\|_{0}=$ $\max _{1 \leq i \leq m}\left\{\sup _{s \in\left[t_{0}-\bar{\tau}, t_{0}\right]_{\top}}\left|\varphi_{i}(s)-\varphi_{i}^{*}(s)\right|\right\}$. In addition, noticing that $e_{\ominus \lambda}\left(t, t_{0}\right) \geq 1$ for $t \in\left[t_{0}-\tau, t_{0}\right]_{\mathbb{T}}$, thus, for any $\epsilon>0$, it is obvious that

$$
\left\|\widetilde{z}\left(t_{0}\right)\right\|<\|\phi\|_{0}+\epsilon
$$

and

$$
\begin{aligned}
\|\widetilde{z}(t)\| & <\left(\|\phi\|_{0}+\epsilon\right) e_{\ominus \lambda}\left(t, t_{0}\right) \\
& <M\left(\|\phi\|_{0}+\epsilon\right) e_{\ominus \lambda}\left(t, t_{0}\right),
\end{aligned}
$$

$$
\forall t \in\left[t_{0}-\tau, t_{0}\right]_{\mathbb{T}} .
$$

We claim that

$$
\|\widetilde{z}(t)\|<M\left(\|\phi\|_{0}+\epsilon\right) e_{\ominus \lambda}\left(t, t_{0}\right), \quad \forall t \in\left(t_{0},+\infty\right)_{\mathbb{T}}
$$

If (110) is not true, then there must be some $t_{1} \in\left(t_{0},+\infty\right)_{\mathbb{T}}$ such that

$$
\begin{aligned}
\left\|\tilde{z}\left(t_{1}\right)\right\| & \geq M\left(\|\phi\|_{0}+\epsilon\right) e_{\ominus \lambda}\left(t_{1}, t_{0}\right), \\
\|\widetilde{z}(t)\| & <M\left(\|\phi\|_{0}+\epsilon\right) e_{\ominus \lambda}\left(t, t_{0}\right),
\end{aligned}
$$


Therefore, there must exist a constant $c \geq 1$ such that

In view of (101), we have

$$
\begin{aligned}
\left\|\tilde{z}\left(t_{1}\right)\right\| & =c M^{*}\left(\|\phi\|_{0}+\epsilon\right) e_{\ominus \lambda}\left(t_{1}, t_{0}\right), \\
\|\tilde{z}(t)\| & <c M^{*}\left(\|\phi\|_{0}+\epsilon\right) e_{\ominus \lambda}\left(t, t_{0}\right),
\end{aligned}
$$

$$
t \in\left[t_{0}-\tau, t_{1}\right)_{\mathbb{T}} .
$$

$$
\begin{aligned}
& \left|\widetilde{z}_{i}\left(t_{1}\right)\right| \\
& =\mid \widetilde{z}_{i}\left(t_{0}\right) \\
& \cdot e_{-\widehat{c}_{i}}\left(t_{1}, t_{0}\right) \int_{t_{0}}^{t} e_{-\widehat{c}_{i}}(t, \sigma(s))\left(\sum _ { j = 1 } ^ { m } \widehat { a } _ { i j } ( s ) \left(\widetilde { f } _ { j } \left(\widetilde{z}_{j}\left(s-\widetilde{\gamma}_{i j}(s)\right)\right.\right.\right. \\
& \left.\left.+\tilde{x}_{j}^{*}\left(s-\widetilde{\gamma}_{i j}(s)\right)\right)-\tilde{f}_{j}\left(\tilde{x}_{j}^{*}\left(s-\widetilde{\gamma}_{i j}(s)\right)\right)\right)+\sum_{j=1}^{m} \sum_{l=1}^{m} \widehat{b}_{i j l}(s)\left(\left[\widetilde{g}_{j}\left(\widetilde{z}_{j}\left(s-\widetilde{\omega}_{i j l}(s)\right)+\widetilde{x}_{j}^{*}\left(s-\widetilde{\omega}_{i j l}(s)\right)\right)\right.\right. \\
& \left.-\widetilde{g}_{j}\left(\widetilde{x}_{j}^{*}\left(s-\widetilde{\omega}_{i j l}(s)\right)\right)\right] \widetilde{g}_{l}\left(\widetilde{z}_{l}\left(s-\widetilde{v}_{i j l}(s)\right)\right. \\
& \left.+\widetilde{x}_{l}^{*}\left(s-\widetilde{v}_{i j l}(s)\right)\right)+\left[\widetilde{g}_{l}\left(\widetilde{z}_{l}\left(s-\widetilde{v}_{i j l}(s)\right)+\widetilde{x}_{l}^{*}\left(s-\widetilde{v}_{i j l}(s)\right)\right)-\widetilde{g}_{l}\left(\widetilde{x}_{l}^{*}\left(s-\widetilde{v}_{i j l}(s)\right)\right)\right] \\
& \left.\left.\cdot \widetilde{g}_{j}\left(\tilde{x}_{j}^{*}\left(s-\widetilde{\omega}_{i j l}(s)\right)\right)\right)\right) \Delta s \mid \\
& \leq e_{-\widehat{c}_{i}}\left(t_{1}, t_{0}\right)\left(\|\phi\|_{0}+\epsilon\right) \\
& +c M e_{\ominus \lambda}\left(t_{1}, t_{0}\right)\left(\|\phi\|_{0}+\epsilon\right) \int_{t_{0}}^{t_{1}} e_{-\widehat{c}_{i}}\left(t_{1}, \sigma(s)\right) \\
& \cdot e_{\lambda}\left(t_{1}, \sigma(s)\right) \times\left(\sum_{j=1}^{m} \widehat{a}_{i j}^{+} \epsilon_{j} e_{\lambda}\left(\sigma(s), s-\widetilde{\gamma}_{i j}(s)\right)+\sum_{j=1}^{m} \sum_{l=1}^{m} \widehat{b}_{i j l}^{+}\left[\varepsilon_{j} N_{l} e_{\lambda}(\sigma(s), s\right.\right. \\
& \left.\left.\left.-\widetilde{\omega}_{i j l}(s)\right)+\varepsilon_{l} N_{j} e_{\lambda}\left(\sigma(s), s-\widetilde{v}_{i j l}(s)\right)\right]\right) \Delta s \\
& \leq e_{-\widehat{c}_{i}}\left(t_{1}, t_{0}\right)\left(\|\phi\|_{0}+\epsilon\right) \\
& +c M e_{\ominus \lambda}\left(t_{1}, t_{0}\right)\left(\|\phi\|_{0}+\epsilon\right) \int_{t_{0}}^{t_{1}} e_{-\hat{c}_{i} \oplus \lambda}\left(t_{1}, \sigma(s)\right) \times\left(\sum_{j=1}^{m} \widehat{a}_{i j}^{+} \epsilon_{j} \exp \left[\lambda\left(\tilde{\gamma}+\sup _{s \in \mathbb{T}} \mu(s)\right)\right]+\sum_{j=1}^{m} \sum_{l=1}^{m} \hat{b}_{i j l}^{+}\right. \\
& \left.\cdot\left[\varepsilon_{j} N_{l} \exp \left[\lambda\left(\widetilde{\omega}+\sup _{s \in \mathbb{\mathbb { T }}} \mu(s)\right)\right]+\varepsilon_{l} N_{j} \exp \left[\lambda\left(\widetilde{v}+\sup _{s \in \mathbb{T}} \mu(s)\right)\right]\right]\right) \Delta s \\
& =c M e_{\ominus \lambda}\left(t_{1}, t_{0}\right)\left(\|\phi\|_{0}+\epsilon\right) \\
& \cdot\left\{\frac{1}{c M} e_{-\widehat{c}_{i} \oplus \lambda}\left(t_{1}, t_{0}\right)+\exp \left[\lambda \sup _{s \in \mathbb{T}} \mu(s)\right] \times\left(\sum_{j=1}^{m} \widehat{a}_{i j}^{+} \epsilon_{j} \exp (\lambda \widetilde{\gamma})+\sum_{j=1}^{m} \sum_{l=1}^{m} \hat{b}_{i j l}^{+}\right.\right. \\
& \left.\left.\cdot\left[\varepsilon_{j} N_{l} \exp (\lambda \widetilde{\omega})+\varepsilon_{l} N_{j} \exp (\lambda \widetilde{v})\right]\right) \times \int_{t_{0}}^{t_{1}} e_{-\widehat{c}_{i} \oplus \lambda}\left(t_{1}, \sigma(s)\right)\right\}
\end{aligned}
$$




$$
\begin{aligned}
& \leq c M e_{\ominus \lambda}\left(t_{1}, t_{0}\right)\left(\|\phi\|_{0}+\epsilon\right) \\
& \cdot\left\{\frac{1}{c M} e_{-\widehat{c}_{i} \oplus \lambda}\left(t_{1}, t_{0}\right)+\exp \left[\lambda \sup _{s \in \mathbb{T}} \mu(s)\right] \times\left(\sum_{j=1}^{m} \widehat{a}_{i j}^{+} \epsilon_{j} \exp (\lambda \widetilde{\gamma})+\sum_{j=1}^{m} \sum_{l=1}^{m} \widehat{b}_{i j l}^{+}\right.\right. \\
& \left.\cdot\left[\varepsilon_{j} N_{l} \exp (\lambda \widetilde{\omega})+\varepsilon_{l} N_{j} \exp (\lambda \widetilde{v})\right]\right) \\
& \left.\times \frac{1-e_{-\widehat{c}_{i} \oplus \lambda}\left(t_{1}, t_{0}\right)}{\widehat{c_{i}}-\lambda}\right\} \\
& \leq c M e_{\ominus \lambda}\left(t_{1}, t_{0}\right)\left(\|\phi\|_{0}+\epsilon\right)\left\{\left[\frac{1}{M}-\frac{\exp \left(\lambda \sup _{s \in \mathbb{T}} \mu(s)\right)}{\widehat{c}_{i}^{-}-\lambda}\left(\sum_{j=1}^{m} \widehat{a}_{i j}^{+} \epsilon_{j} \exp (\lambda \widetilde{\gamma})+\sum_{j=1}^{m} \sum_{l=1}^{m} \widehat{b}_{i j l}^{+}\right.\right.\right. \\
& \left.\left.\cdot\left[\varepsilon_{j} N_{l} \exp (\lambda \widetilde{\omega})+\varepsilon_{l} N_{j} \exp (\lambda \widetilde{v})\right]\right)\right] e_{-\widehat{c}_{\oplus} \oplus \lambda}\left(t_{1}, t_{0}\right)+\frac{\exp \left(\lambda \sup _{s \in \mathbb{T}} \mu(s)\right)}{\widehat{c}_{i}^{-}-\lambda}\left(\sum_{j=1}^{m} \widehat{a}_{i j}^{+} \epsilon_{j} \exp (\lambda \widetilde{\gamma})+\sum_{j=1}^{m} \sum_{l=1}^{m} \widehat{b}_{i j l}^{+}\right. \\
& \left.\left.\cdot\left[\varepsilon_{j} N_{l} \exp (\lambda \widetilde{\omega})+\varepsilon_{l} N_{j} \exp (\lambda \widetilde{v})\right]\right)\right\} \\
& <c M e_{\ominus \lambda}\left(t_{1}, t_{0}\right)\left(\|\phi\|_{0}+\epsilon\right),
\end{aligned}
$$

which contradicts the first equation (112). Therefore, (110) holds. Letting $\epsilon \longrightarrow 0^{+}$leads to

$$
\|\widetilde{z}(t)\| \leq M\|\phi\|_{0} e_{\ominus \lambda}(t, 0), \quad \forall t \in\left(t_{0},+\infty\right)_{\mathbb{T}} .
$$

Hence, the almost periodic solution of system (88) is globally exponentially stable. The proof is complete.

\section{Conclusion}

In this paper, we proposed two types of concepts of almost periodic functions on the quantum time scale and investigated some their basic properties. Moreover, we established a result about the existence and uniqueness of almost periodic solutions of dynamic equations on the quantum time scale by Lyapunov method and gave an equivalent concept of almost periodic functions on the quantum time scale. In addition, as an application, we proposed a class of high-order Hopfield neural network on the quantum time scale and established the existence of almost periodic solutions of the neural networks. Based on the concepts introduced and the results obtained in this paper, we can further study almost periodic problems for dynamic equations on the quantum time scale. For example, we can study the almost periodicity of population dynamical models on the quantum time scale. Furthermore, by using the idea of transformations of (76) and (78), we can give a new concept of almost periodic functions on time scales as follows.
Definition 39. Let $\mathbb{T}$ be a time scale and $\widetilde{\mathbb{T}}$ be an almost periodic time scale defined by Definition 1 and there exists a mapping $\chi: \widetilde{\mathbb{T}} \longrightarrow \mathbb{T}$. A function $f \in C\left(\mathbb{T} \times D, E^{n}\right)$ is called an almost periodic function in $t \in \mathbb{T}$ uniformly in $x \in D$ if, for every $\varepsilon>0$ and for each compact subset $S$ of $D$, there exists a constant $\ell(\varepsilon, S)>0$ such that each interval of length $\ell(\varepsilon, S)$ contains a $\tau(\varepsilon, S) \in \widetilde{\Pi}$ such that

$$
\begin{aligned}
&|f(\chi(t+\tau), x)-f(\chi(t), x)|<\varepsilon, \\
& \forall(t, x) \in \widetilde{\mathbb{T}} \times S,
\end{aligned}
$$

where $\widetilde{\Pi}=\{\tau \in \mathbb{R}: t+\tau \in \widetilde{\mathbb{T}}, \forall t \in \widetilde{\mathbb{T}}\} . \varepsilon$ is called the $\varepsilon$ translation number of $f$.

Obliviously, Definition 39 greatly extends the concept of almost periodic functions on time scales that was introduced in [8] and it unifies the concepts of almost periodic functions on time scales for the cases of $\mathbb{T}=\mathbb{R}, \mathbb{T}=\mathbb{Z}, \mathbb{T}=\overline{q^{\mathbb{Z}}}$ and the case $\mathbb{T}$ is an almost periodic time scale defined by Definition 1 . Similarly, we can give new definitions of almost automorphic functions on time scales. These are our future goals.

\section{Data Availability}

No data were used to support this study. 


\section{Conflicts of Interest}

The author declares that he has no conflicts of interest.

\section{Acknowledgments}

This work is supported by the National Natural Science Foundation of China under Grants nos. 11861072 and 11361072.

\section{References}

[1] H. Bohr, "Zur theorie der fast periodischen funktionen, I," Acta Mathematica, vol. 45, no. 1, pp. 29-127, 1925.

[2] H. Bohr, "Zur theorie der fastperiodischen funktionen, II," Acta Mathematica, vol. 46, no. 1-2, pp. 101-214, 1925.

[3] V. Kac and P. Cheung, Quantum Calculus, Universitext, Springer, New York, NY, USA, 2002.

[4] A. Lavagno, A. M. Scarfone, and P. N. Swamy, "Basic-deformed thermostatistics," Journal of Physics A: Mathematical and General, vol. 40, no. 30, pp. 8635-8654, 2007.

[5] A. Lavagno and P. Narayana Swamy, "q-deformed structures and nonextensive statistics: a comparative study," Physica A: Statistical Mechanics and its Applications, vol. 305, no. 1-2, pp. 310-315, 2002.

[6] M. Bohner and J. G. Mesquita, "Periodic averaging principle in quantum calculus," Journal of Mathematical Analysis and Applications, vol. 435, no. 2, pp. 1146-1159, 2016.

[7] M. Bohner and R. Chieochan, "Floquet theory for q-difference equations," Sarajevo Journal of Mathematics, vol. 8, no. 21, pp. 355-366, 2012.

[8] Y. Li and C. Wang, "Uniformly almost periodic functions and almost periodic solutions to dynamic equations on time scales," Abstract and Applied Analysis, vol. 2011, Article ID 341520, 22 pages, 2011.

[9] Y. Li and C. Wang, "Pseudo almost periodic functions and pseudo almost periodic solutions to dynamic equations on time scales," Advances in Difference Equations, vol. 2012, article 77, 2012.

[10] C. Lizama and J. G. Mesquita, "Almost automorphic solutions of dynamic equations on time scales," Journal of Functional Analysis, vol. 265, no. 10, pp. 2267-2311, 2013.

[11] C. Wang and Y. Li, "Weighted pseudo almost automorphic functions with applications to abstract dynamic equations on time scales," Annales Polonici Mathematici, vol. 108, no. 3, pp. 225-240, 2013.

[12] S. H. Hong and Y. Z. Peng, "Almost periodicity of set-valued functions and set dynamic equations on time scales," Information Sciences, vol. 330, pp. 157-174, 2016.

[13] Y. Li and P. Wang, "Almost periodic solution for neutral functional dynamic equations with Stepanov-almost periodic terms on time scales," Discrete and Continuous Dynamical Systems - Series S, vol. 10, no. 3, pp. 463-473, 2017.

[14] M. Bohner and A. Peterson, Dynamic Equations on Time Scales: An Introduction with Applications, Birkhauser, Boston, Mass, USA, 2001.

[15] M. Bohner and A. Peterson, Advances in Dynamic Equations on Time Scales, Birkhauser, Boston, Mass, USA, 2003.

[16] Y. Li and C. Wang, "Almost periodic functions on time scales and applications," Discrete Dynamics in Nature and Society, vol. 2011, Article ID 727068, 20 pages, 2011. 


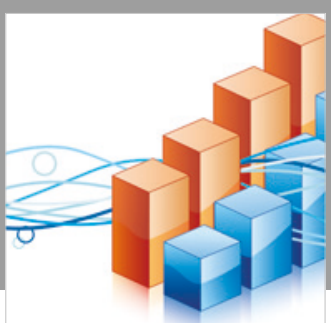

Advances in

Operations Research

\section{-n-m}
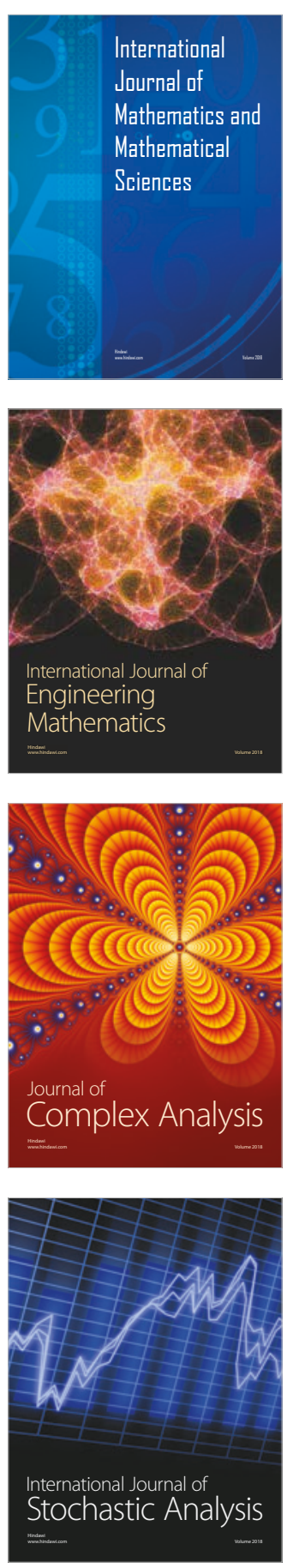
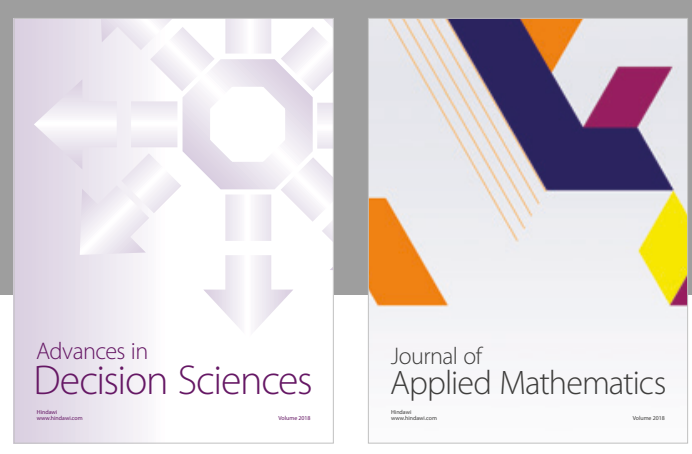

Journal of

Applied Mathematics
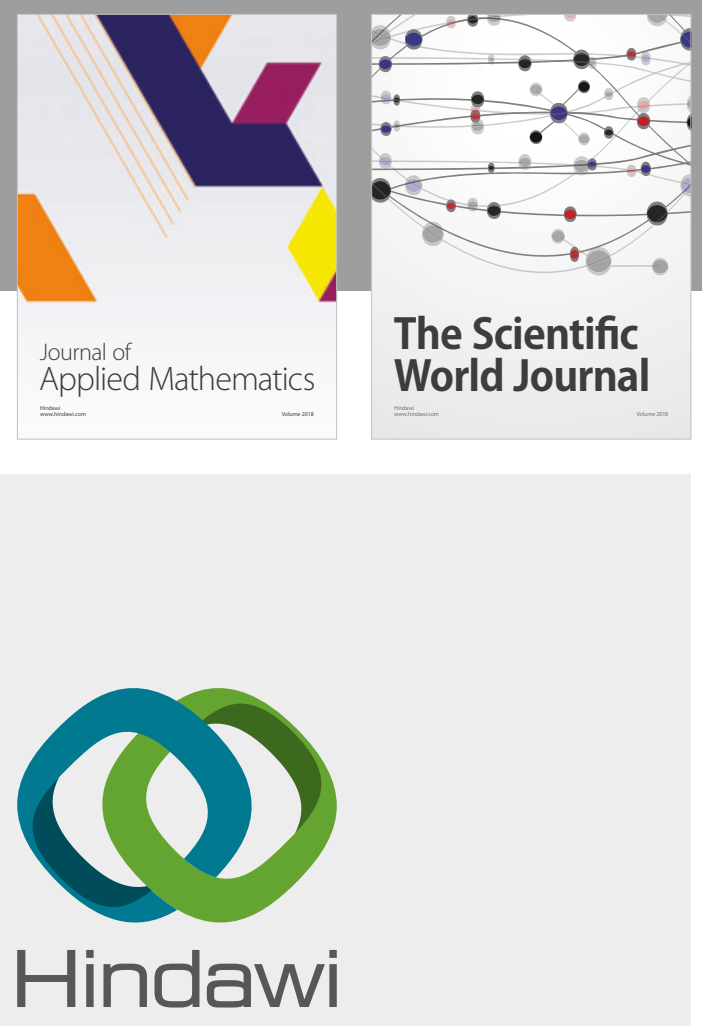

Submit your manuscripts at

www.hindawi.com

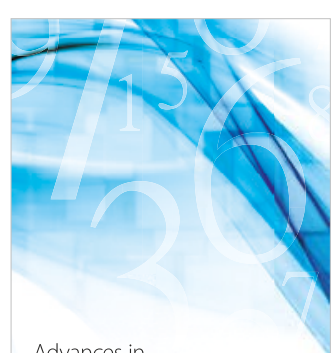

Advances in
Numerical Analysis
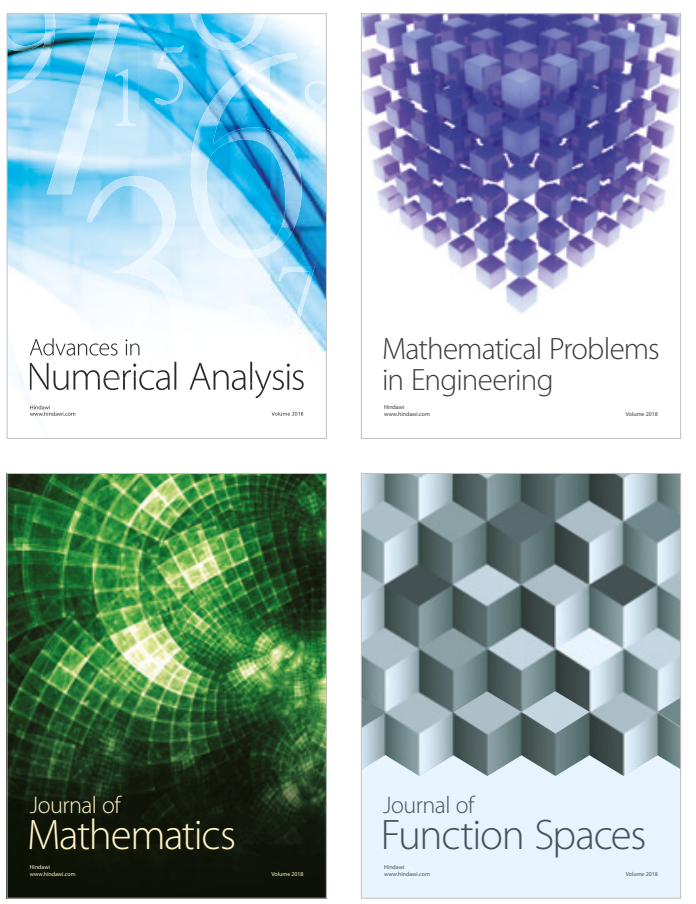

Mathematical Problems in Engineering

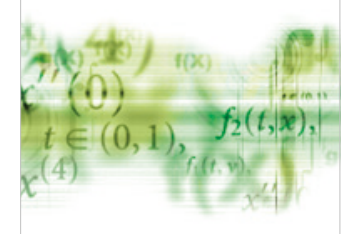

International Journal of

Differential Equations

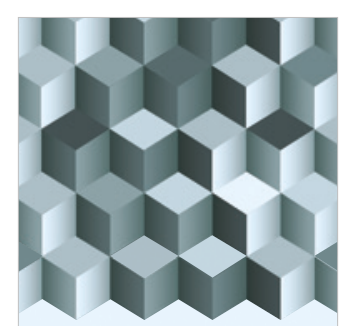

Journal of

Function Spaces

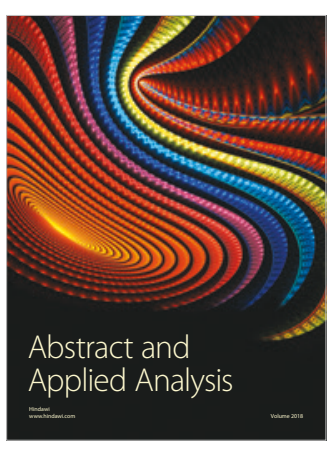

The Scientific

World Journal

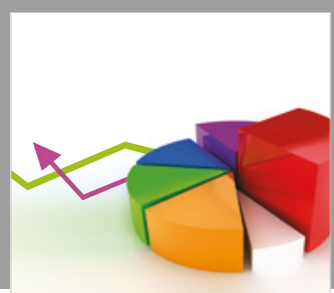

Journal of

Probability and Statistics
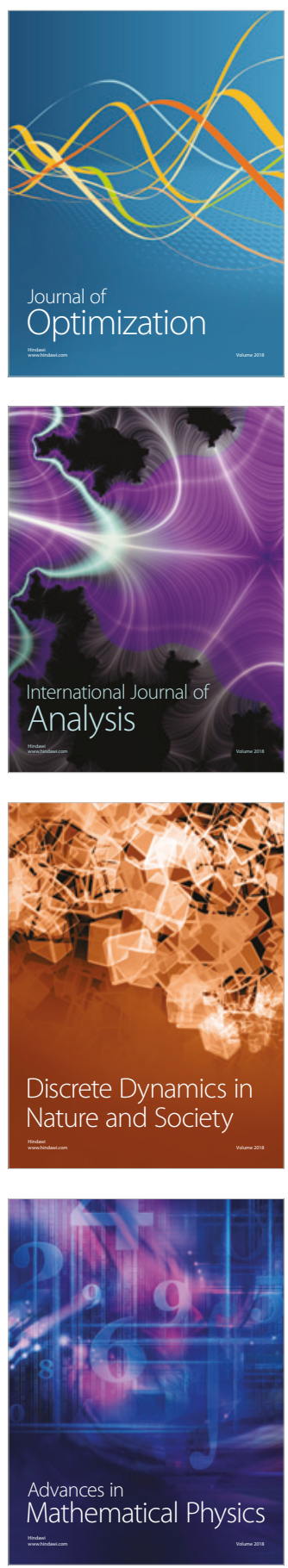